Pacific Journal of Mathematics

2-FACTORIZATION IN FINITE GROUPS 


\title{
2-FACTORIZATION IN FINITE GROUPS
}

\author{
MAKOTO HAYASHI
}

Let $G$ be a finite group, and $S$ be a nonidentity 2-subgroup of $G$. Then, it is naturally conjectured that there exists a nonidentity $N_{G}(S)$-invariant subgroup of $S$, whose normalizer contains all the subgroups $H$ of $G$ with the following properties: $(\alpha) S$ is a Sylow 2-subgroup of $H ;(\beta) H$ does not involve the symmetric group of degree four; and $(\gamma) C_{B}\left(O_{2}(H)\right) \subseteq O_{2}(H)$. The purpose of this paper is to give a partial answer to this problem.

1. Introduction. Suppose $\pi$ is a set of primes, and $X$ is a finite group. Let $\mathscr{D}(X: \pi)$ be the family of all groups $D$ that are involved in $X$ with the following properties; $(\alpha) D$ possesses a normal simple subgroup $E,(\beta) C_{D}(E) \subseteq E$ (that is, $D / C$ induces outer automorphisms of $E$ ), and $(\gamma) D / E$ involves a dihedral group of order $2 p$ for some prime $p(\geqq 5)$ in $\pi$.

THEOREM. Let $\pi$ be a set of primes. Suppose $G$ is a finite group, and $S$ is a nonidentity 2-subgroup of $G$. Assume that for any nonidentity subgroup $T$ of $S$ which is normal in $N_{G}(S)$,

(1) $S$ is normal in some Sylow 2-subgroup of $N_{G}(T)$; and

(2) $\mathscr{D}\left(N_{G}(T) / G_{G}(T): \pi\right)=\dot{\phi}$.

Then there exists a nonidentity subgroup $W(S)$ of $S$ which satisfies the following conditions (a) and (b):

(a) $W(\boldsymbol{S})$ is normal in $N_{G}(S)$; and

(b) $W(S) O(H)$ is normal in $H$ for any solvable $\pi$-subgroup $H$ of $G$ which satisfies the following conditions $(\alpha)$ and $(\beta)$ :

( $\alpha) S$ is a Sylow 2-subgroup of $H$ : and

( $\beta) H$ is $S^{4}$-free, where $S^{4}$ denotes the symmetric group of degree four.

REMARK 1.1. The condition (1) of the theorem is satisfied, whenever $S$ is normal in some Sylow 2-subgroup of $G$.

In general, suppose $p$ is a prime, $G$ is a finite group, and $S$ is a nonidentity $p$-subgroup of $G$. Let $Q d(G, S)$ be the family of all subgroups $H$ of $G$ that satisfy the following conditions: $(\alpha) S$ is a Sylow $p$-subgroup of $H$; and $(\beta) H$ is $p$-constrained, and $p$-stable (if $p=2, S^{4}$-free). Then, what are the relations among the elements of $Q d(G, S)$ ? Furthermore, what are the relations between $G$ and the elements of $Q d(G, S)$ ? These problems were proposed by $G$. Glauberman and J. G. Thompson, and for which amazing progresses 
have been made chiefly by them over the past ten years (c. f. [4], [5], and [6]). The theorem is for $p=2$ a weak analogue of the ZJ-Theorem [3], in which G. Glauberman treated p-groups, namely he succeeded in the Replacement Theorem for $p$-groups with class at most 2, $p$ odd; And he applied it to prove that $Z J(S) \triangleleft H$ for any element $H$ of $Q d(G, S)$. In contrast with this, since we can not find a suitable characteristic subgroup of $S$, we must directly analyze the relations among the solvable elements of $Q d(G, S)$.

The $\S \S 3$ and 4 are used for preliminaries. In the $\S 5$, we get expressions among the solvable elements of $Q d(G, S)$ through the intermediary $S$, and in the $\S 6$, we apply them to some groups involved in $G$. In his paper [5], G. Glauberman defined a new characteristic subgroup $\hat{J}(S)$ for a finite 2-group $S$ which possesses good properties, in particular, in connection with $J_{e}(S)$ and $\Omega_{1} Z(S)$. In this paper, these good properties are exploited which make possible to prove the existence of $W(S)$ which corresponds to $Z J(S)$, but unfortunately, which is not in general characteristic in $S$. We shall treat nonsolvable subgroups in [9].

2. Notation and definition. All groups considered in this paper will be finite. For every finite set $S$, denote the number of elements of $S$ by $|S|$. Let $T$ and $U$ be subsets of $S$. $T \backslash U$ denotes the set of all elements of $T$ that do not belong to $U$. Let $X$ be a finite group, and $Y$ and $Z$ be subsets of $X$. We write $Y \subseteq$ $X(Y \subset X)$ to indicate that $Y$ is a (proper) subgroup of $X$. Let $Y Z=\{y z ; y \in Y, z \in Z\}$, and $Y^{Z}=\left\{z^{-1} y z ; y \in Y, z \in Z\right\} . \quad\langle\cdots ; \cdots\rangle$ denotes the group which is generated by all $\cdots$ such that $\cdots$. Let $[y, z]=y^{-1} z^{-1} y z$ for any pair of elements $y$ and $z$ of $X$, and $[Y, Z]=$ $\langle[y, z] ; y \in Y, z \in Z\rangle . \quad Y \triangleleft X$ if $Y$ is a normal subgroup of $X$. For a finite group $W, X \simeq W$ if $X$ is isomorphic to $W$. $W$ is involved in $X$ if $X$ contains subgroups $X_{1}$ and $X_{2}$ such that $X_{1} \triangleright X_{2}$ and $X_{1} /$ $X_{2} \simeq W$; otherwise $X$ is $W$-free. For a set of primes $\pi, \pi^{\prime}$ denotes the set of all primes which do not belong to $\pi$. We say that $X$ is a $\pi$-group if $\pi$ contains the set of all prime divisors of $|X| . \quad X$ is $\pi$-closed if $X$ possesses a unique maximal $\pi$-subgroup. $X$ is a dihedral group if $X$ is generated by two elements of order 2 .

Suppose $\pi$ is a set of primes. Denote by:

$N_{X}(Y)$ the normalizer of $Y$ in $X$;

$C_{X}(Y)$ the centralizer of $Y$ in $X$;

$Z(X) \quad$ the center of $X$;

$\Omega_{1}(X)$ the subgroup of $X$ which is generated by every element of $X$ that has prime order;

$\Phi(X)$ the Frattini subgroup of $X$, that is, the intersection of all maximal subgroups of $X$; 
$O_{\pi}(X)$ the maximal normal $\pi$-subgroup of $X$, and let $O(X)=$ $O_{2^{\prime}}(X)$;

$O^{-}(X)$ the subgroup of $X$ which is generated by all elements of $X$ whose orders are coprime to any prime in $\pi$;

$F(X)$ the fitting subgroup of $X$, that is, the maximal normal nilpotent subgroup of $X$;

$F_{\infty}(X)$ the maximal normal solvable subgroup of $X$.

$E(X)$ the subgroup of $X$ which is generated by all quasisimple subnormal subgroups of $X$.

We say that $X$ is quasi-simple if $X=[X, X]$ and $X / Z(X)$ is simple.

In addition to the more standard terminology, for a finite group $X$ and a subgroup $S$ of $X$, we say that $X$ is $S$-irreducible if and only if $X=X_{1}$ or $X_{2}$, whenever both $X_{1}$ and $X_{2}$ are subgroups of $X$ which contain $S$ and $X=\left\langle X_{1}, X_{2}\right\rangle$.

In this paper, both the Thompson subgroup and the Glauberman subgroup will play very crucial roles. We define them according to G. Glauberman [6]. Suppose $S$ is a finite 2-group. Let

$d_{e}(S)=\max .\{|A| ; A$ is an elementary Abelian subgroup of $S\}$,

$J_{e}(S)=\langle A$; $A$ ranges over all elementary Abelian subgroups of $S$ with $\left.|A|=d_{e}(S)\right\rangle$, and $\Omega_{1} Z J_{e}(S)=\Omega_{1}\left(Z\left(J_{e}(S)\right)\right)$.

We say that $S^{*}$ is an $E$-group, if $Z\left(S^{*}\right)$ contains every normal elementary Abelian subgroup $V$ of $S^{*}$ which has the following property:

Whenever $R$ is a nonidentity elementary Abelian subgroup of $S^{*} / C_{S^{*}}(V)$, then $\left|V / C_{V}(R)\right|>|R|^{3 / 2}$ and $|[V, R]|>|R|$.

Definition. Let $S$ be a finite 2-group. Then $\widehat{J}(S)=\left\langle S^{*} ; S^{*}\right.$ ranges over all $E$-groups such that $\left.J_{e}(S) \cong S^{*} \leqq S\right\rangle$

and

$$
\Omega_{1} Z \hat{J}(S)=\Omega_{1}(Z(\hat{J}(S))) .
$$

REMARK 1.2. $\widehat{J}(S) \supseteqq J_{e}(S)$ (c.f. [6, Chapter II, Remark 1.1]).

3. Preliminaries and konwn results. In this section, we shall present lemmas which will be frequently quoted and will be used to prove Propositions 3.13 and 17.

Hypothesis A. Suppose $H$ is a finite solvable group, and $S$ is a subgroup of $H$. Assume:

(A.1) $S$ is a Sylow 2-subgroup of $H$; and

(A.2) $H$ is $S^{4}$-free, where $S^{4}$ denotes the symmetric group of 
degree 4.

Theorem 3.1. (G. Glauberman) Suppose $H$ is a finite solvable group, and $S$ is a subgroup of $H$. Assume the pair $(H, S)$ satisfies Hypothesis $A$. Then
(a) $H=C_{H}(Z(S)) N_{H}\left(J_{e}(S)\right) O(H)$;
(b) $H=C_{H}\left(\Omega_{1} Z \hat{J}(S)\right) N_{H}\left(J_{e}(S)\right) O(H)$; and
(c) $H=C_{H}(Z(S)) N_{H}(\hat{J}(S)) O(H)$.

Proof. See [6, Chapter II., Theorem B].

Lemma 3.2. Suppose $S$ is a finite 2-group. Then

(a) $J_{e}(T)=J_{e}(S)$, whenever $J_{e}(S) \subseteq T \subseteq S$;

(b) $\hat{J}(T)=\hat{J}(S)$, whenever $\hat{J}(S) \subseteq T \subseteq S$; and

(c) $\Omega_{1} Z \widehat{J}(T) \supseteqq \Omega_{1} Z \hat{J}(S)$, whenever $J_{e}(S) \subseteq T \cong S$.

Proof. (a) and (b) follow from [6, Chapter II. Lemma 2.1(b) and (d)]. If $T^{*}$ is an $E$-subgroup of $T$, then $T^{*}$ is an $E$-subgroup of $S$ by definition. So, (c) follows from (a).

Lemma 3.3. Suppose $X$ is a finite group, and $V$ is a normal subgroup of $X$. Let $S$ be a Sylow p-subgroup of $X$. Then

(a) $S \cap V$ is a Sylow p-subgroup of $V$;

(b) $S V / V$ is a Sylow p-subgroup of $X / V$;

(c) (Frattini argument) $X=V N_{X}(S \cap V)$; and

(d) $X=O^{p}\left(O^{p^{\prime}}(X)\right) N_{X}(S)$.

Proof. (a), (b) and (c) follow from [8, Theorem 1.3.7 and 8, page 12]. (d) is a special case of (c).

Lemma 3.4. (W. Burnside) Let $p$ be a prime. Suppose $P$ is a finite p-group, and $A$ is a finite group which acts on $P$. Assume that $A$ acts trivially on $P / \Phi(P)$. Then, $\left[P, O^{p}(A)\right]=1$.

Proof. See [8, Theorem 5.1.4, page 174].

Lemma 3.5. (P. Hall) Suppose $p$ is a prime, and $\pi$ is a set of primes which contains $p$. Let $X$ be a finite solvable group, $D_{1}$ and $D_{2}$ be Hall $\pi$-subgroups of $X$, and $S$ be a Sylow p-subgroup of $D_{1}$. Then

(a) $D_{1}$ and $D_{2}$ are conjugate in $X$; and

(b) if $S$ is also a Sylow p-subgroup of $D_{2}$, then there exists an element $x$ of $N_{X}(S)$ such that $D_{1}{ }^{x}=D_{2}$. 
Proof. (a) [follows from [8, Theorem 6.4.1 (ii), page 231]. follows from (a).

LEMMA 3.6. Suppose $X$ is a finite group, and $W$ is a normal subgroup of $X$. Then, $E(W)$ is a central product of uniquely determined quasi-simple groups which are permuted by conjugation of $X$.

Proof. See [7, Lemma (2.1)(a), page 73-74].

LemMa 3.7. Let $r$ be a prime. Suppose $V$ is a finite group, and $H$ is a finite group which acts on $V$. Assume:

(1) $H$ stabilizes a normal series of $V: V \supseteqq V_{1} \supseteq 1$; and

(2) $V_{1}$ is an $r$-group.

Then, $\left[V, O^{r}(H)\right]=1$.

Proof. Let $Q$ be a Sylow $q$-subgroup of $H$, where $q$ is a prime which is distinct from $r$. By (1), $R$ normalizes some Sylow $p$-subgroup of $V$ for any prime divisor $p$ of $|V|$, and centralizes it by [8, Theorem 5.3.2, page 178]. Since $q$ is an arbitrary prime with $q \neq r$, by (2), we get $R \subseteq C_{H}(V) \triangleleft H$, which implies this lemma.

We use the following famous result without notice:

Lemma 3.8. (W. Burnside) Let $X$ be a finite group. Assume that the number of prime divisors of $|X|$ is at most 2 . Then $X$ is solvable.

Proof. See [8, Theorem 4.3.3, page 131].

Lemma 3.9. Suppose $H$ is a finite $\{2,3\}$-group. Then, the following (a) and (b) are equivalent:

(a) $H=0_{3,2,3}(H)$;

(b) $H$ is $S^{4}$-free, where $S^{4}$ denotes the symmetric group of degree four.

Proof. Obviously, (b) follows from (a). Assume (b). Let $X$ be an involved group in $H$ minimal subject to satisfying $H \neq$ $O_{3,2,3}(H)$. Then, $X$ possesses the following properties: $(\alpha) \quad X=$ $O_{2,3,2}(X),(\beta) O_{2}(X)$ is a nonidentity elementary Abelian group, $(\gamma)$ the order of a Sylow 3-subgroup $Q$ of $X$ is 3 , and $(\delta) C_{X}(Q) \cap O_{2}(X)=1$. So, $N_{X}(Q)$ is isomorphic to the symmetric group of degree three, and $O_{2}(X)$ is a four group. This implies that $X \simeq S^{4}$, as required. 
Hence, this lemma is proved.

The next lemma immediately follows from the definitions.

Lemma 3.10. Suppose $H$ is a finite group, and $S$ is a Sylow 2-subgroup of $H$. Then, $H$ possesses $S$-irreducible subgroups $\left\{H_{i}\right.$; $1 \leqq i \leqq t\}$ with a Sylow 2-subgroup $S$ such that $H=\left\langle H_{i} ; 1 \leqq i \leqq t\right\rangle$.

Lemma 3.11. Suppose $H$ is a finite solvable S-irreducible group with a Sylow 2-subgroup $S$. Let $V$ be a normal subgroup of $H$. Then

(a) $H / V$ is $S V / V$-irreducible;

(b) $H$ is a $\{2, r\}$-group for some prime $r$;

(c) $H=O_{2, r, 2}(H)$.

Let $R$ be a Sylow r-subgroup of $H$.

(d) $S \Phi(R) / O_{2}(H) \Phi(R)$ acts irreducibly on $R O_{2}(H) / O_{2}(H) \Phi(R)$;

(e) if $O^{2}(H) \nsubseteq V$, then $S \cap V \triangleleft H$.

In the following statements, we assume that $H$ is not 2-closed.

(f) if $S \leqq V$, then $V=H$, that is, $H=O^{2}(H)$;

(g) if $O^{2}(H) \nsubseteq V$, then $R \cap V \subseteq \Phi(R)$, and $H / V$ involves a dihedral group of order $2 r$.

(h) if $r \neq 3$, one of the following holds:

(h.1) $H$ is r-closed;

(h.2) $H=C_{H I}\left(\Omega_{1} Z(S)\right)=S C_{H}\left(\Omega_{1} Z \widehat{J}(S)\right)$;

(h.3) $H=N_{H H}\left(J_{e}(S)\right)=N_{H}(\hat{J}(S))$;

(h.4) $H=N_{H}\left(J_{e}(S)\right)=C_{H}\left(\Omega_{1} Z(S)\right)$;

(i) if $r \neq 3$, there exists a (possibly trivial) characteristic subgroup $T$ of $S$ which is normal in $H$ and $H / T$ is $r$-closed.

Proof. (a) follows from the definitions.

(b) follows from a theorem of P. Hall [8, Theorem 6.4.1, page 231].

To prove (c), suppose $H \supset \mathrm{O}_{2, r, 2}(H)$. Let $T=S \cap O_{2, r, 2}(H), H_{1}=$ $N_{H}(T)$, and $H_{2}=O_{2, r}(H) S$. Then, $H \supset H_{i} \supseteqq S, 1 \leqq i \leqq 2$, and $H=$ $H_{1} H_{2}$ by the Frattini argument, which contradicts the fact that $H$ is $S$-irreducible.

(d) follows from a theorem of $\mathrm{H}$. Maschke [8, Theorem 3.3.1, page 66].

(e) By the Frattini argument, $H=N_{H}(S \cap V)(V S)$. As $O^{2}(H) \nsubseteq$ $V S, S \subseteq V S \subset H$. Since $H$ is $S$-irreducible, $H=N_{H}(S \cap V)$, as required.

(f) Suppose $O^{2}(H) \nsubseteq V$. By (e), $H=N_{H}(S)$, which contradicts the fact that $H$ is not 2-closed. Hence, $V \supseteqq O^{2}(H) S=H$, as required. 
(g) By (d), $R \cap V \leqq \Phi(R)$. To prove the latter part of (g), we may assume $V \supseteqq O_{2}(H)$. Let $\bar{H}=H / V$. Then, a theorem of R. Baer [8, Theorem 3.8.2. page 105] implies that $\bar{H}$ possesses involutions $\bar{x}$ and $\bar{y}$ such that $\langle\bar{x}, \bar{y}\rangle$ is not a 2-group, which implies (g).

To prove (h), assume $H \neq O_{r}(H) S$; then, by Theorem 3.1, $H=$ $C_{H}=(Z(S)) N_{H}\left(J_{e}(S)\right)=C_{H}\left(\Omega_{1} Z \hat{J}(S)\right) N_{H}\left(J_{e}(S)\right)=C_{H}\left(\Omega_{1} Z(S)\right) N_{H}(\widehat{J}(S))$. Since $H$ is $S$-irreducible, we get (h).

(i) Let $T$ be a characteristic subgroup of $S$ maximal subject to satisfying $T \triangleleft H$. By Theorem 3.1, $\bar{H}=C_{\bar{H}}(Z(\bar{S})) N_{\bar{H}}\left(J_{e}(\bar{S})\right)(O(\bar{H}) \bar{S})$. Then we conclude $\bar{H}=O(\bar{H}) \bar{S}$ by (a) and the maximality of $T$.

Lemma 3.12. Let $r$ be a prime with $r \geqq 5$. Suppose $D$ is a finite group, $\left\{D_{i} ; 1 \leqq i \leqq n\right\}$ are normal subgroups of $D, S$ is a 2subgroup of $D$, and $H$ is a $\{2, r\}$-subgroup of $D$ with a Sylow 2subgroup S. Assume:

(1) $S$ is normal in some Sylow 2-subgroup of $D$;

(2) $H$ is an $S$-irreducible group which is not 2-closed;

(3) $O^{2}(H) \subseteq D_{1} D_{2} \cdots D_{n}$;

(4) $\left[D_{i}, D_{j}\right]=1$ for all $i ; 1 \leqq i \neq j \leqq n$;

(5) $D_{i} \cap<D_{j}$; $1 \leqq j \neq i \leqq n>i s$ a 2-group for each $i$; $1 \leqq$ $i \leqq n$.

Then there exist subgroups $\left\{K_{i} ; 1 \leqq i \leqq n\right\}$ of $D$ which satisfy the following conditions:

(a) $K_{i}$ is a $\{2, r\}$-group with a Sylow 2-subgroup $S, 1 \leqq i \leqq n$;

(b) $O^{2}\left(K_{i}\right) \leqq D_{i}$ for all $i ; 1 \leqq i \leqq n$;

(c) $\left\langle K_{i} ; 1 \leqq i \leqq n\right\rangle$ is a $\{2, r\}$-group with a Sylow 2-subgroup $S$, and $H \subseteq\left\langle K_{i} ; 1 \leqq i \leqq n\right\rangle$;

(d) thhere exists a subgroup $T$ of $S$ which satisfies the following conditions:

(d.1) $T$ is normal in $\left\langle N_{D}(S), K_{i} ; 1 \leqq i \leqq n\right\rangle$; and

(d.2) $\left\langle K_{i} ; 1 \leqq i \leqq n\right\rangle / T$ is r-closed.

For any subgroup $J$ of $S$,

(e) if $O_{2}(H) \supseteqq J$, then $O_{2}\left(K_{i}\right) \supseteqq J$ for all $i ; 1 \leqq i \leqq n$;

(f) if $O^{2}(H) \subseteq\left[O_{2, r}(H), J\right]$, then $O^{2}\left(K_{i}\right) \subseteq\left[O_{2, r}\left(K_{i}\right), J\right]$ for all $i$; $1 \leqq i \leqq n$;

(g) if $H$ is r-closed, then $\left\langle K_{i} ; 1 \leqq i \leqq n\right\rangle$ is r-closed.

Proof. In this proof, for any subgroup $X$ of $D$, we let

$$
\begin{gathered}
X_{i}^{*}=\left\{x_{i} ; x_{1} x_{2} \cdots x_{n} \in X \cap D_{1} D_{2} \cdots D_{n} \text { and } x_{i} \in D_{i}, 1 \leqq i \leqq n\right\}, \\
1 \leqq i \leqq n .
\end{gathered}
$$

Since $D_{i} \triangleleft D$, it is easily verified that for any subgroup $X$ of $D$, 
$(3.12 .1)$

$$
N_{D}\left(X_{i}^{*}\right) \supseteqq N_{D}(X) \text { for all } i ; 1 \leqq i \leqq n .
$$

For another subgroup $Y$ of $D$, by (4),

(3.12.2) $\left[X \cap D_{1} D_{2} \cdots D_{n}, Y \cap D_{1} D_{2} \cdots D_{n}\right] \subseteq\left[X_{i}^{*}, Y_{i}^{*}\right] ; 1 \leqq i \leqq n ，$

and

(3.12.3) $\left[X_{i}^{*}, Y \cap D_{1} D_{2} \cdots D_{n}\right]=\left[X_{i}^{*}, Y_{i}^{*}\right]$ for all $i$; $1 \leqq i \leqq n$.

Suppose $O^{2}(H) \subseteq D_{i}$ for some $i ; 1 \leqq i \leqq n$. By Lemma 3.11(i), there exists a subgroup $T$ of $S$ such that $T$ is normal in $\left\langle N_{D}(S), H\right\rangle$ and $H / T$ is $r$-closed. Hence, setting $K_{i}=H$ and $K_{j}=S$ for other $j ; 1 \leqq j \neq i \leqq n$, we can easily verify that all our assertions hold. Thus, we may assume that:

$$
O^{2}(H) \nsubseteq D_{i} \text { for all } i ; 1 \leqq i \leqq n .
$$

So, by Lemma 3.11(e),

$$
S \cap D_{i} \triangleleft H \text { for all } i ; 1 \leqq i \leqq n .
$$

By (3.12.1) and (5),

(3.12.6) $\mathrm{O}_{2}(H)_{i}^{*}$ is a 2-group which is normalized by $H$ for all

$$
i ; 1 \leqq i \leqq n \text {. }
$$

By (1), there exists a Sylow 2-subgroup $U$ of $D$ in which $S$ is normal. Since $U \subseteq N_{D}(S) \subseteq N_{D}\left(S_{i}^{*}\right)$ and $O_{2}(H)_{i}^{*} \subseteq S_{i}^{*}$, we obtain that $O_{2}(H)_{i}^{*} \subseteq U$ for all $i ; 1 \leqq i \leqq n$. Since $S \triangleleft U,\left[O_{2}(H)_{i}^{*}, S\right] \subseteq$ $S \cap D_{i}$ for all $i ; 1 \leqq i \leqq n$. Hence, by (3.12.5) and (3.12.6), $S \leqq$ $C_{H}\left(O_{2}(H)_{i}^{*} /\left(S \cap D_{i}\right)\right) \triangleleft H$ for all $i ; 1 \leqq i \leqq n$. So, by (2) and Lemma $3.11(\mathrm{f})$,

(3.12.7) $H=C_{H}\left(O_{2}(H)_{i}^{*} /\left(S \cap D_{i}\right)\right)$ for all $i ; 1 \leqq i \leqq n$.

Let $T=\left\langle S \cap D_{i} ; 1 \leqq i \leqq n\right\rangle$. By (3.12.5),

$$
T \triangleleft\left\langle N_{D}(S), H\right\rangle \text {. }
$$

Let $R$ be a Sylow $r$-subgroup of $H$. Then, by (3.12.2), (3.12.3) and (3.12.7),

$(3.12 .9)$

$$
\begin{aligned}
{\left[O_{2}(H), R\right] } & =\left[O_{2}(H), R, R\right] \\
& \subseteq\left[O_{2}(H) \cap\left(D_{1} D_{2} \cdots D_{n}\right), R\right] \\
& \cong\left[\left\langle O_{2}(H)_{i}^{*} ; 1 \leqq i \leqq n\right\rangle, R\right] \\
& \leqq\left\langle\left[O_{2}(H)_{i}^{*}, R\right] ; 1 \leqq i \leqq n\right\rangle \\
& \leqq\left\langle S \cap D_{i} ; 1 \leqq i \leqq n\right\rangle \\
& \subseteq T .
\end{aligned}
$$


So, by (2) and Lemma 3.11(c).

$H / T$ is $r$-closed.

By (3), (3.12.2), (3.12.3) and (3.12.5), $\left[T, R_{i}^{*}\right]=\left[S \cap D_{i}, R_{i}^{*}\right]=[S \cap$ $\left.D_{i}, R\right] \subseteq S \cap D_{i} \subseteq T$. Hence, $R_{i}^{*}$ normalizes $T$, so that

$$
O_{r}\left(R_{i}^{*}\right) \text { normalizes } T \text { for all } i ; 1 \leqq i \leqq n \text {. }
$$

Then, by (3.12.1), (3.12.10) and the Frattini argument,

$$
\begin{gathered}
S \subseteq N_{H}(R T) \subseteq N_{H}(R) T \subseteq N_{H}\left(R_{i}^{*}\right) T \subseteq N_{H}\left(O_{r}\left(R_{i}^{*}\right) T\right) \\
\text { for all } i ; 1 \leqq i \leqq n
\end{gathered}
$$

It follows that $S O_{r}\left(R_{i}^{*}\right)$ is a $\{2, r\}$-group with a Sylow 2-subgroup $S$ for all $i ; 1 \leqq i \leqq n$. Let $K_{i}=S O_{r}\left(R_{i}^{*}\right), 1 \leqq i \leqq n$. Now, we will check that $K_{i} ; 1 \leqq i \leqq n$ satisfy all the conclusions of this lemma.

(a) is proved in the above argument.

Since $O^{2}\left(K_{i}\right)=\left\langle O_{r}\left(R_{i}^{*}\right)^{x} ; x \in K_{i}\right\rangle \leqq D_{i}, 1 \leqq i \leqq n$, we get (b). By (4) and (5), $R_{i}^{*}$ is $r$-closed, $1 \leqq i \leqq n$, so that $H=S R \subseteq S\left\langle O_{r}\left(R_{i}^{*}\right)\right.$; $1 \leqq i \leqq n\rangle=\left\langle K_{i} ; 1 \leqq i \leqq n\right\rangle$. Since $\left[O_{r}\left(R_{i}^{*}\right), O_{r}\left(R_{j}^{*}\right)\right]=1,1 \leqq i \neq j \leqq$ $n$, it follows that $\left\{K_{i} ; 1 \leqq i \leqq n\right\}$ is a $\{2, r\}$-group with a Sylow 2subgroup $S$. Thus (c) is proved.

By (3.12.8), $T \triangleleft N_{D}(S)$. Then, (d, 1) follows from (3.12.11), and (d.2) follows from (3.12.12). Thus, (d) is proved.

Let $J$ be a subgroup of $S$. Since $J$ normalizes $O^{2}(H)$, we get:

$$
\left[O^{2}(H), J\right]_{i}^{*}=\left[O^{2}(H)_{i}^{*}, J\right] \text { for all } i ; 1 \leqq i \leqq n .
$$

Suppose $J \subseteq O_{2}(H)$. Then by (d), $\left[O^{2}(H), J\right] \subseteq T$. By (d) and (3.12.13), $\left[O^{2}(H)_{i}^{*}, J\right] \subseteq T_{i}^{*}, 1 \leqq i \leqq n$. It follows that

$$
\left[O_{r}\left(R_{1}^{*}\right), J\right]\left[O_{r}\left(R_{2}^{*}\right), J\right] \cdots\left[O_{r}\left(R_{n}^{*}\right), J\right]
$$

is a 2-group, which implies (e).

Suppose $O^{2}(H) \subseteq\left[O_{2, r}(H), J\right]$. Since $H=O_{2, r, 2}(H)$, we have that $R \leqq\left[O^{2}(H), J\right]$. Hence, $O_{r}\left(R_{i}^{*}\right) \subseteq\left[O_{r}\left(R_{i}^{*}\right), J\right] \subseteq\left[O_{2, r}(H), J\right], 1 \leqq i \leqq n$, which implies (f).

Finally, suppose $H$ is $r$-closed. Then, $\left[O^{2}(H), T\right]=1$. By (3.12.2), $\left[O^{2}\left(K_{i}\right), T\right]=1,1 \leqq i \leqq n$. By (d), it follows that $K_{i}$ is $r$-closed for all $i ; 1 \leqq i \leqq n$, which proves $(\mathrm{g})$. Hence this lemma is proved.

Proposition 3.13. Let $r$ be a prime with $r \geqq 5$. Suppose $D$ a finite group, $V$ is a normal subgroup of $D,\left\{D_{i} ; 1 \leqq i \leqq n\right\}$ are normal subgroups of $D$ all of which contain $V, S$ is a 2-subgroup of $D$, and $H$ is a $\{2, r\}$-subgroup of $D$ with a Sylow 2-subgroup $S$. Assume: 
(1) $S$ is normal in some Sylow 2-subgroup of $D$;

(2) $H$ is an S-irreducible group which is not 2-closed;

(3) $O^{2}(H) \subseteq D_{1} D_{2} \cdots D_{n}$;

(4) $V$ is 2-closed with $O_{2}(V)=S \cap V$.

Let $\bar{X}=X V / V$ for any subgroup $X$ of $D$.

(5) $\left[\bar{D}_{i}, \bar{D}_{j}\right]=1$ for all $i, j ; 1 \leqq i \neq j \leqq n$;

(6) $\bar{D}_{i} \cap\left\langle\bar{D}_{j} ; 1 \leqq j \neq i \leqq n\right\rangle$ is a 2-group for each $i ; 1 \leqq i \leqq n$. Then there exist subgroups $\left\{L_{i} ; 1 \leqq i \leqq t\right\}$ of $D$ which satisfy the following conditions:

(a) $L_{i}$ is an S-irreducible $\{2, r\}$-group with a Sylow 2-subgroup $S$ for all $i ; 1 \leqq i \leqq t$

(b) $\left\langle L_{i} ; 1 \leqq i \leqq t\right\rangle$ is a $\{2, r\}$-group with a Sylow 2-subgroup $S$, and $H \subseteq\left\langle L_{i} ; 1 \leqq i \leqq t\right\rangle$;

(c) there exists a subgroup $T$ of $S$ which is normal in $\left\langle N_{D}(S)\right.$, $\left.L_{i} ; 1 \leqq i \leqq t\right\rangle$, and $\left\langle L_{i} ; 1 \leqq i \leqq t\right\rangle / T$ is r-closed.

(d) for each $L_{i}, 1 \leqq i \leqq t, O^{2}\left(L_{i}\right)$ is contained in some $D_{j}, 1 \leqq$ $j \leqq n$

(e) if $H=N_{H}\left(J_{e}(S)\right)$, then $L_{i}=N_{L_{i}}\left(J_{e}(S)\right)$ for all $i ; 1 \leqq i \leqq t$;

(f) if $H \neq N_{H}\left(J_{e}(S)\right)$, then $L_{i} \neq N_{L_{i}}\left(J_{e}(S)\right), 1 \leqq i \leqq t$; and

(g) if $H / V \cap S$ is r-closed, then $L_{i} / V \cap S$ is r-closed, $1 \leqq i \leqq t$.

Proof. In this proof, let $\bar{X}=X V / V$ for any subgroup $X$ of D. By Lemma 3.12 , there exist subgroups $\left\{\bar{K}_{i} ; 1 \leqq i \leqq n\right\}$ of $\bar{D}$ which satisfy all the conclusions of Lemma 3.12. Let $F_{1}$ be a Hall $\{2, r\}$-subgroup of the pre-image of $\left\{\bar{K}_{i} ; 1 \leqq i \leqq n\right\}$ in $D$ which contains $H$. Then, by (4) and Lemma 3.12(c),

$$
F_{1} \text { is a }\{2, r\} \text {-group with a Sylow 2-subgroup }
$$

$$
S, H \subseteq F_{1} \text { and } \bar{F}_{1}=\left\langle\bar{K}_{i} ; 1 \leqq i \leqq n\right\rangle \text {. }
$$

By Lemma $3.12(\mathrm{~d})$, there exists a subgroup $\bar{T}$ of $\bar{S}$ such that:

$$
\bar{T} \triangleleft\left\langle N_{\bar{D}}(\bar{S}), \bar{F}_{1}\right\rangle \text { and } \bar{F}_{1} / \bar{T} \text { is } r \text {-closed. }
$$

Let $T$ be the intersection of $S$ and the pre-image of $\bar{T}$ if $\bar{H}$ is not $r$-closed; otherwise, $T=S \cap V$. Then, by (2) and Lemma 3.11(e),

$$
H=N_{H}(T) \text {. }
$$

Let $F_{2}=N_{F_{1}}(T)$. Then, by (3.13.3), Lemma 3.12(c) and (g), (3.13.4) $H \subseteq F_{2}, T \triangleleft F_{2}, F_{2} / T$ is $r$-closed, and $\bar{F}_{2}=\left\langle\bar{K}_{i} ; 1 \leqq i \leqq n\right\rangle$.

Finally, let $F=N_{F_{2}}\left(J_{e}(S)\right)$ if $H=N_{H}\left(J_{e}(S)\right)$; otherwise, let $F=$ $\left.\left[O_{2, r}\left(F_{2}\right), J_{e}(S)\right)\right] S . \quad B y(2)$ and Lemma 3.11(d), if $H \neq N_{H}\left(J_{e}(S)\right)$, then $O^{2}(H) \subseteq\left[O_{2, r}(H), J_{e}(S)\right]$. So, in either case that $H=N_{H}\left(J_{e}(S)\right)$ or $H \neq N_{H}\left(J_{e}(S)\right)$, 


$$
H \subseteq F .
$$

By (3.13.4), Lemma 3.12(e) and (f),

$$
T \triangleleft F \text { and } F / T \text { is } r \text {-closed; and }
$$

$$
\bar{F}=\left\langle\bar{K}_{i} ; 1 \leqq i \leqq n\right\rangle \text {. }
$$

By Lemma 3.12(g),

(3.13.8) if $H / V \cap S$ is $r$-closed, then $F / V \cap S$ is $r$-closed.

By (3.13.7),

$$
F=\left\langle\left(F \cap D_{i}\right) S ; 1 \leqq i \leqq n\right\rangle .
$$

Since $S$ is a Sylow 2-subgroup of $\left(F \cap D_{i}\right) S$, by Lemma 3.10,

(3.13.10) each $\left(F \cap D_{i}\right) S$ is generated by $S$-irreducible subgroups with a Sylow 2-subgroup $S, 1 \leqq i \leqq n$.

Therefore, there exist subgroups $\left\{L_{i} ; 1 \leqq i \leqq t\right\}$ of $F$ which satisfy the following conditions:

$$
L_{i} \text { is an } S \text {-irreducible group with a Sylow 2-subgroup }
$$

$$
S, 1 \leqq i \leqq t
$$

(3.13.12) for each $L_{i}, 1 \leqq i \leqq t, O^{2}\left(L_{i}\right)$ is contained in some

$$
D_{j}, 1 \leqq j \leqq n \text {. }
$$

Furthermore, by (3.13.9),

$$
\left\langle L_{i} ; 1 \leqq i \leqq t\right\rangle=F .
$$

Then, we may assume that:

$$
F \supset\left\langle L_{i} ; 1 \leqq i \neq j \leqq t\right\rangle \text { for any } j ; 1 \leqq j \leqq t .
$$

Now, we check that $\left\{L_{i} ; 1 \leqq i \leqq t\right\}$ satisfy all the conclusions of this lemma. Since $F$ is a $\{2, r\}$-group, (a) follows from (3.13.11), and (b) follows from (3.13.5) and (3.13.13). Since $T \triangleleft N_{D}(S)$, (c) follows from (3.13.6). (d) follows from (3.13.12). And, (g) follows from (3.13.8).

To prove (e), suppose $H=N_{H}\left(J_{e}(S)\right)$. Then $F=N_{F}\left(J_{e}(S)\right)$, so that $L_{i}=N_{L_{i}}\left(J_{e}(S)\right)$ for all $i ; 1 \leqq i \leqq t$, which proves (e). Next, suppose $H \neq N_{H}\left(J_{e}(S)\right)$. Then by definition,

$$
F=\left[O_{2, r}(F), J_{e}(S)\right] S .
$$

Let $\quad L_{i} \neq N_{L_{i}}\left(J_{e}(S)\right), 1 \leqq i \leqq s$, and $\quad L_{j}=N_{L_{j}}\left(J_{e}(S)\right), s+1 \leqq j \leqq t$. Then, $F=\left\langle N_{F}\left(J_{e}(S)\right), L_{i} ; 1 \leqq i \leqq s\right\rangle$. By (3.13.15), $F=\left[O_{2, r}(F), J_{e}(S)\right] S=$ 
$\left\langle L_{i} ; 1 \leqq i \leqq s\right\rangle$. So, by (3.13.14), $s=t$. Thus, we conclude that $L_{i} \neq N_{L_{i}}\left(J_{e}(S)\right)$ for all $i ; 1 \leqq i \leqq t$, which proves (f). Hence this lemma is proved.

HYPothesis B.I. Suppose $M$ is a finite group, $W$ is a normal subgroup of $M$, and $Y$ is a subgroup of $W$. Assume:

(B.1) $W=E(W)=E_{1} \times \cdots \times E_{r}$, where $E_{k}$ is a non-Abelian simple group with $E_{k} \simeq E_{1}, 1 \leqq k \leqq r ;$ and

(B.2) $\quad E_{k} \nsubseteq Y$ for all $k ; 1 \leqq k \leqq r$.

Let $\Lambda=\{1,2, \cdots, r\}$. We identity $E_{k}$ with the element $k$ of $\Lambda$, $1 \leqq k \leqq r$. Let $\Gamma$ be the following family of subsets of $\Lambda$ :

$\Gamma=\left\{\left\{k_{1}, \cdots, k_{s}\right\} ;\left\{k_{1}, \cdots, k_{s}\right\}\right.$ is minimal under inclusion such that $\left.Y \cap\left(E_{k_{1}} \times \cdots \times E_{k_{s}}\right) \neq 1\right\}$. We say that $s$ is the length of $\left\{k_{1}, \cdots, k_{s}\right\}$. Let $\pi_{k}$ be the projection mapping from $W$ to $E_{k}, 1 \leqq k \leqq r$.

Proposition 3.14. Assume Hypothesis B.I. Assume also that: Then

(B.0) whenever $1 \leqq k \leqq r, \pi_{k}(Y)=E_{k}$.

(a) for any distinct elements $\left\{k_{1}, \cdots, k_{s}\right\}$ and $\left\{j_{1}, \cdots, j_{t}\right\}$ of $\Gamma$, $\left\{k_{1}, \cdots, k_{s}\right\} \cap\left\{j_{1}, \cdots, j_{t}\right\}=\varnothing$;

(b) $|Y|=\left|E_{1}\right|^{|\Gamma|}$;

(c) $|Y|^{2} \leqq|W|$;

(d) if equality holds in (c), then

(d.1) $r$ is even;

for suitable renumbering $A$,

(d.2) $\quad \Gamma=\{\{k, k+r / 2\} ; 1 \leqq k \leqq r / 2\}$;

(d.3) $Y=\prod_{k=1}^{r / 2}\left(Y \cap\left(E_{k} \times E_{k+r / 2}\right)\right)$, and $Y \cap\left(E_{k} \times E_{k+r / 2}\right) \simeq E_{1}$, $1 \leqq k \leqq r / 2$.

(e) conversely, if the lengths of all elements of $\Gamma$ are equal to 2 , then equality holds in (c);

(f) $W$ centralizes $\left(\bigcap_{k=1}^{r} N_{M}\left(E_{k}\right)\right) \cap C_{M}(Y)$.

Proof. Take an arbitrary element $\left\{k_{1}, \cdots, k_{s}\right\}$ of $\Gamma$. We may assume that $\left\{k_{1}, \cdots, k_{s}\right\}=\{1, \cdots, s\}$, renumbering if necessary.

Let $Y_{0}=Y \cap\left(\coprod_{k=1}^{s} E_{k}\right)$, and let $\psi_{k}$ be the projection mapping from $Y_{0}$ to $E_{k}, 1 \leqq k \leqq s$.

By minimal nature of $\{1, \cdots, s\}$,

$\operatorname{Ker} \psi_{k}=1$, and $\operatorname{Im} \psi_{k} \neq 1,1 \leqq k \leqq s$

Since $Y_{0} \triangleleft Y$, by (3.14.1), (B.1) and (B.0),

(3.14.2) $\quad \operatorname{Im} \psi_{k}=\left\langle\left(\operatorname{Im} \psi_{k}\right)^{r}\right\rangle=\left\langle\left(\operatorname{Im} \psi_{k}\right)^{E} k\right\rangle=E_{k}, 1 \leqq k \leqq s$.

By (3.14.1) and (3.14.2), we conclude that $i_{k}$ is an isomorphism 
for all $k ; 1 \leqq k \leqq s$.

Therefore,

$$
Y_{0} \simeq E_{1}
$$

To prove (a), take distinct elements $\left\{k_{1}, \cdots, k_{s}\right\}$ and $\left\{j_{1}, \cdots, j_{t}\right\}$ of $\Gamma$.

Let $Y_{1}=Y \cap\left(\prod_{i=1}^{s} E_{k_{i}}\right)$, and $Y_{2}=Y \cap\left(\prod_{i=1}^{t} E_{j_{i}}\right)$. By minimality of $\left\{k_{1}, \cdots, k_{s}\right\}$ and $\left\{j_{1}, \cdots, j_{t}\right\}, Y_{1} \cap Y_{2}=1$. Since $Y_{1} \triangleleft Y$ and $Y_{2} \triangleleft Y$,

$$
\left[Y_{1}, Y_{2}\right]=1 \text {. }
$$

Suppose that $\left\{k_{1}, \cdots, k_{s}\right\} \cap\left\{j_{1}, \cdots, j_{t}\right\} \neq \varnothing$, and take $h$ in this intersection. By (3.14.4) and (B.0), $1=\left[\Psi_{h}\left(Y_{1}\right), \Psi_{h}\left(Y_{2}\right)\right]=\left[E_{h}, E_{h}\right]$, which contradicts (B.1), and (a) is proved. Then (B.0) implies that $\Lambda=\cup \gamma$ (disjoint union), where $\gamma$ ranges over all the elements of $\Gamma$. Then (3.14.3) yields (b), and (c) follows from (b) and (B.2).

Assume equality holds in (c). By (c), $r$ is even, and we may assume that $\Gamma=\{\{k, k+r / 2\} ; 1 \leqq k \leqq r / 2\}$. By (a) and (3.14.3),

$$
Y=\prod_{k=1}^{r / 2}\left(Y \cap\left(E_{k} \times E_{k+r / 2}\right)\right) \simeq \prod_{k=1}^{r / 2} E_{k},
$$

which proves (d).

(e) follows from (b) and (B.0). Finally, to prove (f), take an arbitrary subscript $k$ and an arbitrary element $x_{k}$ of $E_{k}$. By (B.0), $Y$ possesses an element $y$ such that $y=x_{1} \cdots x_{k} \cdots x_{r}$, where $x_{i} \in E_{i}$, $1 \leqq i \leqq r . \quad$ Let $K=\left(\bigcap_{k=1}^{r} N_{M}\left(E_{k}\right)\right) \cap C_{M}(Y)$. Then $1=[K, y]=[K$, $\left.x_{1}\right] \cdots\left[K, x_{k}\right] \cdots\left[K, x_{r}\right]$, and $\left[K, x_{i}\right] \leqq E_{i}, 1 \leqq i \leqq r$. Therefore $\left[K, x_{k}\right]=$ 1 , which implies (f).

Hypothesis B.II: Assume Hypothesis B.I. Let $q$ be an odd prime. Suppose $N$ is a subgroup of $M$ which normalizes $Y, H$ is a subgroup of $N$, and $S$ is a Sylow 2-subgroup of $H$. Assume also that:

(B.3) $\quad N=\left\langle H, N_{N}(S)\right\rangle$;

(B.4) there exists a subgroup $T$ of $S$ such that

(B.4.1) $T\left(S \cap O_{2}(N)\right)=S$, and

(B.4.2) $[R, T] \subseteq Y$ for any $S$-invariant $q$-subgroup $R$ of $W$;

(B.5) there exists an $S$-invariant $q$-subgroup $Q$ of $W$ such that $\left\langle\pi_{1}(Q)^{x} ; x \in N\right\rangle=W$.

Only for convenience to state and prove the following Proposition 3.15 , we need one more hypothesis:

Hypothesis B.III: Assume Hypothesis B.II. Assume also that: (B.0) whenever $1 \leqq k \leqq r, \pi_{k}(Y)=E_{k}$; and 
(B.6) $|Y|^{2}=|W|$.

Proposition 3.15. Assume Hypothesis B.II. Then $O^{2}\left(O^{2}(H)\right)$ normalizes each $E_{k}, 1 \leqq k \leqq r$. Moreover, at least one of the following (a) or (b) holds:

(a) $T$ normalizes each $E_{k}, 1 \leqq k \leqq r$; or

(b) Hypothesis B.III is satisfied, and $T$ fixes each element of $\Gamma$.

Proof. The proof is separated into seven steps. In the first step, we will note down the matters which will be needed for the development of the proof.

Step 1. (1.a) $O_{2}(N) \cap S \triangleleft N$;

(1.b) $\left\langle\pi_{1}(Q)^{x} ; x \in N_{N}\left(E_{1}\right)\right\rangle=E_{1}$;

(1.c) $N$ acts transitively on $\Lambda$;

(1.d) $N$ induces a permutation of $\Gamma$, and if Hypothesis (B.0) is satisfied, $N$ acts transitively on $\Gamma$; and

(1.e) if Hypothesis (B.0) is satisfied and the length of some element of $\Gamma$ is equal to 2, then Hypothesis B.III is satisfied.

Proof of Step 1. Since $S$ is a Sylow 2-subgroup of $H, O_{2}(N) \cap$ $S \triangleleft H$. Since $O_{2}(N) \cap S \triangleleft N_{N}(S)$, (B.3) yields (1.a).

By (B.5), $\quad E_{1}=E_{1} \cap\left\langle\pi_{1}(Q)^{x} ; x \in N\right\rangle=\left\langle\pi_{1}(Q)^{x} ; x \in N_{N}\left(E_{1}\right)\right\rangle$, which proves (1.b). Again, by (B.5), (1.c) follows from (1.b).

Since $N$ leaves invariant $Y, N$ induces a permutation of $\Gamma$. By Proposition 3.14(a) and (1.c), $\Gamma=\left\{\gamma^{x} ; x \in N\right\}$, which proves (1.d). Suppose Hypothesis (B.0) is satisfied and the length of some element of $\Gamma$ is equal to 2. Then by (1.d), the lengths of all elements of $\Gamma$ are equal to 2. (1.e) follows from (1.d) and Proposition 3.14(e).

Step 2. Fix $k=1,2, \cdots, r$. Let $R_{k}$ be a $q$-subgroup of $E_{k k}$. Assume:

(1) $N_{S}\left(E_{k}\right)$ normalizes $R_{k}$;

(2) $\left\langle R_{k}^{x} ; x \in N_{N}\left(E_{k}\right)\right\rangle=E_{k}$; and

(3) $T$ does not normalize $E_{k}$.

Then

(2.a) Hypothesis B.III is satisfied; and

(2.b) whenever $x \in T$ and $k^{x} \neq k,\left\{k, k^{x}\right\}$ lies in $\Gamma$.

Proof of Step 2. Let $R=\left\langle R_{k}^{y} ; y \in S\right\rangle$. By (1), $R$ is an S-invariant $q$-subgroup of $W$. By (3), there exists an element $x$ of $T$ such that $E_{k}^{x} \neq E_{k}$. By (B.4.2), 
$(3.15 .1)$

$$
\pi_{k}(Y) \supseteqq \pi_{k}([R, T]) \supseteqq \pi_{k}([R, x])=\pi_{k}\left(R_{k}\right)=R_{k},
$$

and

$$
Y \cap\left(E_{k} \times E_{k}^{x}\right) \supseteqq Y \cap\left[R_{k}, x\right] \neq 1 .
$$

Since $Y$ is $N$-invariant, by (2) and (3.15.1), $\pi_{k}(Y) \supseteqq\left\langle R_{k}^{y} ; y \in N_{N}\left(E_{k}\right)\right\rangle=$ $E_{k}$. By (1.c),

$$
\pi_{j}(Y)=E_{j}, 1 \leqq j \leqq r
$$

By (3.15.2),

$$
\left\{k, k^{x}\right\} \text { lies in } \Gamma \text {. }
$$

Then (2.a) follows from (3.15.3), (3.15.4) and (1.e). Since $x$ is an arbitrary element of $T$ such that $k^{x} \neq k$, (2.b) follows from (3.15.4). Hence this lemma is proved.

Let $\left\{1^{x} ; x \in S\right\}=\{1,2, \cdots, s\}$, renumbering if necessary.

Step 3. Assume $T$ does not normalize some $E_{k}, 1 \leqq k \leqq s$. Then

(3.a) Hypothesis B.III is satisfied; and

(3.b) whenever $x \in T$ and $k^{x} \neq k,\left\{k, k^{x}\right\}$ lies in $\Gamma$.

Proof of Step 3. By (B.5) and (1.b), $N_{S}\left(E_{k}\right)$ normalizes $\pi_{k}(Q)$ and $\left\langle\pi_{k}(Q)^{y} ; y \in N_{N}\left(E_{k}\right)\right\rangle=E_{k}$. Thus, this step follows from Step 2.

Let $\left\{1^{x} ; x \in O_{2}(N) \cap S\right\}=\{1,2, \cdots, w\}$, and $\left\{1^{x} ; x \in H\right\}=\{1,2, \cdots, h\}$, renumbering if necessary.

Step 4. Assume $w \neq s$. Then

(4.a) Hypothesis B.III is satisfied;

(4.b) $2 w=s$;

(4.c) $h / w$ is even.

Proof of Step 4. Since $O_{2}(N) \cap S \triangleleft H$ by (1.a), for any element $x$ of $H$,

$$
\{1,2, \cdots, w\}^{x} \text { is an }\left(O_{2}(N) \cap S\right) \text {-orbit; and }
$$

Since $w \neq s$, by (B.4.1), there exists an element $t$ of $T$ such that $\{1,2, \cdots, w\} \cap\{1,2, \cdots, w\}^{t}=\varnothing$. Let $k^{t}=k+w, 1 \leqq k \leqq w$. Then we get (4.a) from (3.a). Then by (3.b),

$$
\{k, k+w\} \text { lies in } \Gamma \text { for all } k ; 1 \leqq k \leqq w .
$$


Now, to prove (4.b), by (B.4.1) and (3.15.5), we may only show that:

(3.15.8) for any element $x$ of $T,\{1,2, \cdots, w\}^{x} \cong\{1,2, \cdots, 2 w\}$.

Assume that $\{1,2, \cdots, w\}^{x} \neq\{1,2, \cdots, w\}$ for some element $x$ of $T$. Since $t$ is an arbitrary element of $T$ with $\{1,2, \cdots, w\}^{t} \neq\{1,2, \cdots$, $w\}$ in (3.15.7), we obtain that $\left\{k, k^{x}\right\}$ lies in $\Gamma$ for all $k ; 1 \leqq k \leqq w$. Then by Proposition $3.14(\mathrm{a}), k^{x}=k^{t}=k+w$ for all $k ; 1 \leqq k \leqq w$, which implies (3.15.8). Hence, (4.b) is proved. To prove (4.c), it is enough to show that:

$$
\begin{gathered}
\{1,2, \cdots, 2 w\} \cap\{1,2, \cdots, 2 w\}^{x}=\varnothing \text { or }\{1,2, \cdots, 2 w\} \\
\text { for any element } x \text { of } H .
\end{gathered}
$$

Assume that for some element $x$ of $H,\{1,2, \cdots, 2 w\} \cap\{1,2, \cdots, 2 w\}^{x} \neq$ $\varnothing$, and take $j$ in this intersection. Then by (3.15.7), $\left\{j, j+w^{*}\right\}$ lies in $\Gamma$, where $w^{*}=w$ if $1 \leqq j \leqq w$, and $w^{*}=-w$ if $w+1 \leqq$ $j \leqq 2 w$. So, by (1.d), $\left\{j^{x^{-1}},\left(j+w^{*}\right)^{x^{-1}}\right\}$ lies in $\Gamma$. Since $j^{x^{-1}}$ lies in $\{1,2, \cdots, 2 w\}$, by (3.15.7) and Proposition 3.14(a), $\left(j+w^{*}\right)^{x^{-1}}$ lies in $\{1,2, \cdots, 2 w\}$. It follows that $\{1,2, \cdots, w\} \cap\{1,2, \cdots, 2 w\}^{x} \neq \varnothing$ and $\{w+1, w+2, \cdots, 2 w\} \cap\{1,2, \cdots, 2 w\}^{x} \neq \varnothing$. Then, (3.15.9) follows from (3.15.6). Hence (4.c) is proved.

Step 5. $\quad N_{S}\left(E_{1}\right)$ is a Sylow 2-subgroup of $N_{H}\left(E_{1}\right)$.

Proof of Step 5. In this proof, for any natural number $n$, let $n_{2}$ be the highest power of 2 which divides $n$.

Let $v=h / w$.

By Step 4, we have that either $s=w$ or $s=2 w$ and $v$ is even. Thus,

$$
s \leqq w v_{2}
$$

Since $\left|N_{H}\left(E_{1}\right)\right|_{2}=|H|_{2} / h_{2}=|S| / w v_{2}=\left|N_{S}\left(E_{1}\right)\right|\left(s / w v_{2}\right)$, (3.15.10) implies that $\left|N_{H}\left(E_{1}\right)\right|_{2} \leqq\left|N_{S}\left(E_{1}\right)\right|$, which proves this step.

Step 6. Assume $T$ does not normalize some $E_{k}, 1 \leqq k \leqq h$. Then

(6.a) Hypothesis B.III is satisfied;

(6.b) $h$ is even; and

(6.c) for some suitable renumbering, whenever $1 \leqq k \leqq h / 2$, then $\{k, k+h / 2\}$ lies in $\Gamma$, and $\{k, k+h / 2\}^{t}=\{k, k+h / 2\}$ for any element $t$ of $T$. 
Proof of Step 6. Let $V$ be a Sylow 2-subgroup of $N_{H}\left(E_{k}\right)$ which contains $N_{S}\left(E_{k}\right)$. By Step 5, there exists an element $z$ of $H$ such that $N_{S}\left(E_{1}\right)^{z}=V$ and $E_{1}^{z}=E_{k}$. Then by $(1 . \mathrm{b}),\left\langle\pi_{1}\left(Q^{z}\right)^{y} ; y \in\right.$ $\left.N_{N}\left(E_{k}\right)\right\rangle=E_{k}$. By (B.5), $V$ normalizes $\pi_{1}(Q)^{z}=\pi_{k}\left(Q^{z}\right)$, so that $N_{S}\left(E_{k}\right)$ normalizes $\pi_{k}\left(Q^{z}\right)$. Hence, (3.a) yields (6.a).

Let $x$ be an element of $T$ such that $k^{x} \neq k$. By (3.b), $\left\{k, k^{x}\right\}$ lies in $\Gamma$. By (1.d) and Proposition 3.14(a),

$\left\{k, k^{x}\right\}^{y}$ lies in $\Gamma$ for any element $y$ of $H$, and $\{1,2, \cdots, h\}=\bigcup_{y}\left\{k, k^{x}\right\}^{y}$ (disjoint union), where

$y$ ranges over all the elements of $H$.

This implies (6.b). Moreover, renumbering if necessary, we may assume: $\{k, k+h / 2\}$ lies in $\Gamma, 1 \leqq k \leqq h / 2$. To prove the final assertion of this step, take an element $t$ of $T$ and an element $\{k, k+h / 2\}$ of $\Gamma, 1 \leqq k \leqq h / 2$. Then by the above, $k=k^{t}$ or $\left\{k, k^{t}\right\}$ lies in $\Gamma$, so that $\{k, k+h / 2\} \cap\{k, k+h / 2\}^{t} \neq \varnothing$. Then by (3.15.11), $\{k, k+h / 2\}^{t}=\{k, k+h / 2\}$, as required.

Step 7. $O^{2}\left(O^{2^{\prime}}(H)\right)$ normalizes each $E_{k}, 1 \leqq k \leqq h$.

Proof of Step 7. First, assume that $T$ normalizes each $E_{k}, 1 \leqq$ $k \leqq h$. Let $H_{0}=\bigcap_{k=1}^{h} N_{H}\left(E_{k}\right)$. By (B.4.1) and (1.a), $S=\left(O_{2}(N) \cap\right.$ $S) T \subseteq\left(O_{2}(N) \cap S\right) H_{0} \triangleleft H$. Thus, $O^{2}\left(O^{\prime}(H)\right) \subseteq H_{0}$, as required. So, we may assume that $T$ does not normalize some $E_{k}, 1 \leqq k \leqq h$. Then, Step 6 shows that Hypothesis B.III is satisfied, and we may assume that $\{k, k+h / 2\}$ lies in $\Gamma, 1 \leqq k \leqq h / 2$. Let $H_{1}=\{x \in H$; $\{k, k+h / 2\}^{x}=\{k, k+h / 2\}$ for all $\left.k ; 1 \leqq k \leqq h / 2\right\}$. Then by (B.4.1), (1.a) and (6.c), $S=\left(O_{2}(N) \cap S\right) T \subseteq\left(O_{2}(N) \cap S\right) H_{1} \triangleleft H$. Thus,

$$
O^{2}\left(O^{2^{\prime}}(H)\right) \subseteq H_{1} \text {. }
$$

Since the lengths of all elements of $\Gamma$ are equal to 2 and $O^{2}\left(O^{2}(H)\right)$ is generated by elements of odd order, $O^{2}\left(O^{2}(H)\right)$ must fix each $E_{k}, 1 \leqq k \leqq h$. Hence this step is proved.

Proof of Proposition 3.15. By Lemma 3.3(d),

$$
H=O^{2}\left(O^{2}(H)\right) N_{H}(S) .
$$

So, by (B.3),

$$
N=\left\langle O^{2}\left(O^{2}(H)\right), N_{N}(S)\right\rangle .
$$

Take an element $x$ of $N_{N}(S)$. Let $Q^{*}=\left\langle\pi_{1}(Q)^{x y} ; y \in S\right\rangle, k=1^{x}$, and $\gamma$ be an element of $\Gamma$ which contains $k$. By (B.5), $Q^{*}$ is an 
$S$-invariant $q$-subgroup of $W$, and by (1.b), $\left\langle\pi_{k}\left(Q^{*}\right)^{y} ; y \in N_{N}\left(E_{k}\right)\right\rangle=E_{k}$. Then, replacing $E_{k}$ by $E_{1}$, all the assumptions of Hypothesis B.II are satisfied. Then Step 7 and (6.c) show that: $O^{2}\left(O^{2}(H)\right)$ normalizes $E_{k}$; and $T$ fixes $k$, or Hypothesis B.III is satisfied and $T$ fixes $\gamma$. Since $x$ is an arbitrary element of $N_{N}(S)$, by (1.c) and (3.15.12), $N_{N}(S)$ acts transitively on $\Lambda$. Then, the above argument implies this lemma.

Lemma 3.16. Let $p$ be a prime with $p \geqq 5$. Suppose $M$ is a finite group, $W$ is a normal subgroup of $M$, and $H$ is a subgroup of $M$ with a Sylow 2-subgroup S. Assume:

(1) $W=E(W)=E_{1} \times E_{2} \times \cdots \times E_{r}$, where $E_{k}$ is non-Abelian simple, $1 \leqq k \leqq r$;

(2) $H$ is an S-irreducible $\{2, p\}$-group which is not 2-closed;

(3) $H=O_{2}(H)\left(\bigcap_{k=1}^{r} N_{H}\left(E_{k}\right)\right)$; and

Then

(4) $\mathscr{D}(W H: p)=\varnothing$.

$$
O^{2}(H) \subseteq W C_{M}(W)
$$

Proof. Let $H_{0}=\bigcap_{k=1}^{r} N_{H}\left(E_{k}\right)$.

Then, $H_{0} \triangleleft H$.

By (3),

$$
\begin{gathered}
H_{0} / O_{2}\left(H_{0}\right) \text { is isomorphic to a homomorphic image of } \\
H, \text { and } O^{2}(H) \subseteq H_{0} .
\end{gathered}
$$

Suppose $O^{2}\left(H_{0}\right) \nsubseteq E_{k} C_{M}\left(E_{k}\right)$ for some $k ; 1 \leqq k \leqq r$. Then by Lemma $3.11(\mathrm{~g}), H_{0} /\left(H_{0} \cap E_{k} C_{M}\left(E_{k}\right)\right)$ involves a dihedral group of order $2 p$, which contradicts (4). Hence, $O^{2}(H) \subseteq O^{2}\left(H_{0}\right) \subseteq \bigcap_{k=1}^{r} E_{k} C_{M}\left(E_{k}\right) \subseteq W C_{M}(W)$, as required.

Proposition 3.17. Let $p$ be a prime with $p \geqq 5$. Assume Hypothesis B.II. Further assume that:

(1) $H$ is an S-irreducible $\{2, p\}$-group which is not 2-closed;

(2) $\mathscr{D}(W H: p)=\varnothing$.

Then, $O^{2}(H) \subseteq W C_{M}(W)$.

Proof. By (1) and Lemma 3.11(f), $H=O^{2}(H)$. Then by Proposition 3.15 and $3.14(\mathrm{~d}), O^{2}(H)$ normalizes each $E_{k}, 1 \leqq k \leqq r$. Moreover, at least one of the following holds:

$(\alpha) T$ normalizes each $E_{k}, 1 \leqq k \leqq r$; or

$(\beta)$ for some suitable renumbering of $\{1,2, \cdots, r\}, T$ normalizes each $Y \cap\left(E_{k} \times E_{k+r / 2}\right)$, and $Y \cap\left(E_{k} \times E_{k+r / 2}\right) \simeq E_{1}$ for all $k ; 1 \leqq k \leqq r / 2$. Assume $(\alpha)$ holds. Then by (B.4.1), $S=\left(S \cap O_{2}(N)\right) T \subseteq O_{2}(H) T \subseteq$ 
$O_{2}(H)\left(\bigcap_{k=1}^{r} N_{H}\left(E_{k}\right)\right) \triangleleft H$. By (1) and Lemma 3.11(f),

$$
O_{2}(H)\left(\bigcap_{k=1}^{r} N_{I I}\left(E_{k}\right)\right)=H .
$$

Then by Lemma 3.16, $O^{2}(H) \subseteq W C_{M}(W)$, as required. Next, assume $(\beta)$ holds. Then by the same argument as above, we have that $O_{2}(H)\left(\bigcap_{k=1}^{r / 2} N_{H}\left(Y \cap\left(E_{k} \times E_{k+r / 2}\right)\right)\right)=H$. Then by Lemma 3.16 (with $Y$ in place of $W), O^{2}(H) \subseteq Y C_{M}(Y)$. Let $x$ be any element of $O^{2}(H)$. Then, we may let $x=x_{1} x_{2}$, where $x_{1} \in Y$ and $x_{2} \in C_{M}(Y)$. By Lemma 3.14(f),

$$
x_{2}=x_{1}^{-1} x \in\left(\bigcap_{k=1}^{r} N_{M}\left(E_{k}\right)\right) \cap C_{M}(Y) \leqq C_{M}(W) .
$$

Since $x$ is an arbitrary element of $O^{2}(H)$, we conclude that $O^{2}(H) \subseteq$ $W C_{M}(W)$. Hence this lemma is proved.

4. Preliminaries in the minimal situation. From now on, we shall prove the theorem by way of contradiction. Let $\pi$ be a set of primes. Suppose that $G$ is a finite group, and $S$ is a nonidentity 2-subgroup of $G$. Assume that $(\pi, G, S)$ satisfies all the assumptions of the theorem, but violates the conclusions of the theorem. Take $G$ of minimal order and, subject to this condition, take $S$ of minimal order.

Lemma 4.1. Let $T$ and $T_{0}$ be subgroups of $S$. Assume:

(1) $1 \subset T_{0} \subseteq T \subset S$;

(2) $T \triangleleft N_{G}(S)$; and

(3) $T_{0} \triangleleft N_{G}(T)$.

Then

(a) $T_{0} \triangleleft N_{G}(S)$.

So by the assumption (1) of the theorem, there exists a Sylow 2subgroup $U$ of $N_{G}\left(T_{0}\right)$ in which $S$ is normal. Then,

(b) $T \triangleleft U$;

(c) $\mathscr{D}\left(N_{G}\left(T_{0}\right) / C_{G}\left(T_{0}\right): \pi\right)=\varnothing$;

(d) there exists a unique nonidentity subgroup $W(T)$ of $T$ maximal subject to satisfying the following condition:

(d.1) $W(T) \triangleleft N_{G}(T)$; and

(d.2) $W(T) O(H) \triangleleft H$ for any $\pi$-subgroup $H$ of $G$ such that the pair $(H, T)$ satisfies Hypothesis $A$.

Let $N=N_{G}(T)$. Suppose that $S_{0} / T$ is a nonidentity subgroup of $S / T$ which is normal in $N_{N / T}(S / T)$. Then,

(e) $S_{0} / T \triangleleft U / T$;

(f) $\mathscr{D}\left(N_{N / T}\left(S_{0} / T\right) / C_{N / T}\left(S_{0} / T\right): \pi\right)=\varnothing$;

(g) there exists a unique nonidentity subgroup $W(S / T)$ of $S / T$ 
maximal subject to satisfying condition:

(g.1) $W(S / T) \triangleleft N_{N / T}(S / T)$;

(g.2) $\quad W(S / T) O(H / T) \triangleleft H / T$ for any $\pi$-subgroup $H / T$ of $N / T$ such that the pair $(H / T, S / T)$ satisfies Hypothesis $A$.

Proof. Obvious.

Notation. In the following discussion, without notice, we shall use the following notation: for any subgroup $T$ of $S$ such that $1 \subset T \subset S$ and $T \triangleleft N_{G}(S)$, we denote by $W(T)$ (or $W(S / T)$ ) the subject to satisfying the conclusions of (d) (or $(\mathrm{g})$ ) of Lemma 4.1.

DEFINITION. Let:

$$
\mathfrak{F}_{G}(S)=\{H \subseteq G \text {; the pair }(H, S) \text { satisfies Hypothesis } A\},
$$

and

$$
\widetilde{\mho}_{G}(S)=\left\{H \in \mathfrak{F}_{G}(S) ; H \text { is } S \text {-irreducible }\right\} .
$$

For a subfamily $\Re$ of $\mathfrak{F}_{G}(S)$, we define $O_{S}(\Re)$ to be the largest subgroup of $S$ that satisfies the following conditions (a) and (b):

(a) $O_{S}(\Omega) \triangleleft N_{G}(S)$; and

(b) $K=N_{K}\left(O_{S}(\Omega)\right) O(K)$ for any element $K$ of $\Re$.

For simplicity, let $O_{S}(K)=O_{S}(\{K\})$ for any element $K$ of $\widetilde{F}_{G}(S)$. Whenever $1 \subset O_{S}(\Re)$, we define: $W(\Omega: 0)=1, W(\Omega: 1)=O_{S}(\Re)$, and let $W(\Omega: \nu+1)$ be the pre-image of $W(S / W(\Omega: \nu))$ in $S, \nu=1,2, \cdots$.

Then, we introduce the function $f_{\text {se }}$ which is defined on $\Omega$ as the following: for any element $K$ of $\Re$,

$$
f_{\S}(K)=\max .\{\nu ; K \triangleright W(\Re: \nu), \quad 0 \leqq \nu \leqq \rho\},
$$

where $\rho$ denotes the nonnegative integer such that $W(\Re: \rho)=S$ and $W(\Omega: \rho-1) \subset S$.

REMARK 4.1. Whenever $W(\Re: \nu) \subset S, \quad W(\Omega: \nu) \subset W(\Re: \nu+1)$, because $W(S / W(\Re: \nu)) \neq 1$.

Lemma 4.2. Let $K$ be an element of $\widetilde{\mho}_{G}(S)$. Then the following (a) and (b) are equivalent:

(a) $K$ is 2-closed or 2'-closed;

(b) $O_{S}(K)=S$.

Proof. Assume (a). Then, $K=N_{K}(S) O(K)$, which shows (b). Conversely, assume (b). Then, $K=N_{K}(S) O(K)=N_{K}(S)(O(K) S)$. Since $K$ is $S$-irreducible, it follows that $K=N_{K}(S)$ or $K=O(K) S$, 
which proves (a).

Lemma 4.3. Suppose $\Re$ is a subfamily of $\mathfrak{\mho}_{G}(S)$, none of whose elements are 2'-closed. Let $K$ be an element of $\Re$. Assume that $1 \subset O_{S}(\Re)$. Let $\nu=f_{\Omega}(K)$. Then

(a) $O_{S}(\Re) \triangleleft\left\langle N_{G}(S), H \in \Re\right\rangle$, and $O_{S}(\Omega)=S \cap O_{2}\left(\left\langle N_{G}(S), H \in \Re\right\rangle\right)$;

(b) $\nu \geqq 1$, and $f_{\Omega}(L)=1$ for some element $L$ of $\Omega$;

(c) $K / W(\Re: \nu)$ is 2'-closed;

(d) $K / O_{S}(K)$ is 2'-closed;

(e) $O_{S}(K) \supseteqq V$, whenever $V \triangleleft N_{G}(S)$ and $V \subseteq O_{2}(K)$;

(f) $\left[O^{2}(K), O_{S}(K)\right] \subseteq O_{S}(L)$, whenever $L \in \Re$ and $f_{\varepsilon}(L) \geqq \nu$.

Proof. (a) Let $H$ be an arbitrary element of $\Omega$. Since $H$ is an $S$-irreducible group which is not $2^{\prime}$-closed,

$$
H=N_{H}\left(O_{S}(\Re)\right) O(H)=N_{H}\left(O_{S}(\Re)\right)(O(H) S)=N_{H}\left(O_{S}(\Re)\right),
$$

which implies the former part of (a).

Let $T=S \cap O_{2}\left(\left\langle N_{G}(S), H \in \Re\right\rangle\right)$. By the former part of (a), $O_{S}(\Re) \subseteq T$. On the other hand, for any element $L$ of $\Re, L \triangleright L \cap$ $O_{2}\left(\left\langle N_{G}(S), H \in \Re\right\rangle\right)=T$, which shows $T \subseteq O_{S}(\Re)$. Hence, (a) is proved.

By (a) and the definition of $f_{\mathfrak{\varepsilon}}$, we obtain that $\nu \geqq 1$. Suppose that $f_{\Omega}(L)>1$ for any element $L$ of $\Omega$. Then by definition, $W(\Omega: 2) \subset$ $W(\Re: 1)$, which is a contradiction. Hence (b) is proved. To prove (c), we may assume $S \supset W(\Re: \nu)$; otherwise, (c) is obvious. By (a) and definition, $K \triangleright W(\Omega: \nu)$. Let $\bar{X}=X W(\Omega: \nu) / W(\Omega: \nu)$ for any subgroup $X$ of $K$. By Lemma 3.11(a), $\bar{K}$ is $\bar{S}$-irreducible. Suppose $\bar{K}$ is not 2 -closed. By induction,

$$
\bar{K}=N_{\bar{K}}(W(\bar{S})) O(\bar{K})=N_{\bar{K}}(W(\bar{S}))(O(\bar{K}) \bar{S})=N_{\bar{K}}(W(\bar{S})) .
$$

Hence, $K \triangleright W(\Omega: \nu+1) \supset W(\Omega: \nu)$, which contradicts $\nu=f_{s}(K)$. Thus, (c) is proved.

(d): By (a), $K \triangleright O_{S}(K)$. By maximality of $O_{S}(K), O_{S}(K) \supseteqq$ $W(\Omega: \nu)$. Then, (c) yields (d).

(e) By (d), $\left[K, V O_{S}(K)\right] \cong V O_{S}(K)$. Since $V O_{S}(K) \triangleleft N_{G}(S)$, $V \subseteq V O_{S}(K) \subseteq O_{S}(K)$ by maximality of $O_{S}(K)$, which proves (e).

(f) Let $\mu=f_{\AA}(L)$. By (c), $\left[O^{2}(K), O_{S}(K)\right] \subseteq W(\Re: \nu) \subseteq W(\Re: \mu) \subseteq$ $O_{S}(L)$, which proves (f), and this lemma.

LeMma 4.4. Let $\Re$ be a subfamily of $\mathfrak{F}_{G}(S)$. Assume that there exists a subgroup $T$ of $S$ which satisfies the following conditions:

(1) $1 \subset T \subset S$;

(2) $T \triangleleft N_{G}(S)$; and 
Then

(3) $\left[O^{2}(K), O_{S}(K)\right] \subseteq T$ for any element $K$ of $\Re$.

$$
O_{S}(\Re) \supset 1
$$

Proof. Let $\Re=\left\{K_{i} ; 1 \leqq i \leqq t\right\}$, and $L_{i}=O^{2}\left(K_{i}\right) T, 1 \leqq i \leqq t . \quad$ By (3) and Lemma $4.3(\mathrm{~d}), L_{i}$ is a group with a Sylow 2-subgroup $T$, and $O\left(L_{i}\right) \subseteq O\left(K_{i}\right)$ for all $i ; 1 \leqq i \leqq t$. By induction, $W(T) \supset 1$. Since $L_{i}=N_{L_{i}}(W(T)) O\left(L_{i}\right), K_{i}=N_{K_{i}}(W(T)) O\left(K_{i}\right)$ for all $i ; 1 \leqq i \leqq t$. Therefore, we conclude that $1 \subset W(T) \subseteq O_{S}(\Re)$, as required. Hence this lemma is proved.

LEMma 4.5. $O_{S}\left(\mathfrak{F}_{G}(S)\right)=O_{S}\left(\widetilde{\mho}_{G}(S)\right)=1$.

Proof. Since $(G, S)$ is a counterexample to the theorem,

$$
O_{S}\left(\mathfrak{F}_{G}(S)\right)=1 .
$$

So, we may show only that $O_{S}\left(\mathfrak{F}_{G}(S)\right)=O_{S}\left(\mathfrak{\mho}_{G}(S)\right)$. To prove this equation, let $T=O_{S}\left(\mathfrak{\mho}_{G}(S)\right)$. Obviously, $O_{S}\left(\mathfrak{F}_{G}(S)\right) \leqq T$. To prove the opposite inclusion, take an element $K$ of $\mathfrak{S}_{G}(S)$. By Lemma $3.10, \dot{K}=\left\langle K_{i} ; 1 \leqq i \leqq t\right\rangle$ for some suitable elements $\left\{K_{i} ; 1 \leqq i \leqq\right.$ $t\}$ of $\mathfrak{F}_{G}(S)$. Then $K_{i}=N_{K_{i}}(T) O\left(K_{i}\right)$, and $O\left(K_{i}\right) \subseteq O(K)$ for all $i$; $1 \leqq i \leqq t$. Therefore, $K=N_{K}(T) O(K)$. Since $K$ is an arbitrary element of $\xi_{G}(S)$, it follows that $T \subseteq O_{S}\left(\xi_{G}(S)\right)$, which proves this lemma.

5. Properties of elements of $\mathscr{C} \mathscr{L}(G)$.

Definition. Let $m$ be the natural number defined as follows: $O_{S}(\Re) \supset 1$ for any subfamily $\Re$ of $\widetilde{F}_{G}(S)$ with $|\Re|<m$;

and

$O_{S}(\Re)=1$ for some subfamily $\Re$ of $\mathfrak{\mho}_{G}(S)$ with $|\Re|=m$.

REMARK 5.1. Such a natural number $m$ exists by Lemma 4.5.

Define the class of subfamilies $\mathscr{C}_{\mathscr{L}} \mathscr{L}(G)$ of $\widetilde{\mho}_{G}(S)$ as follows:

$$
\mathscr{C} \mathscr{P}(G)=\left\{\Re \subseteq \widetilde{F}_{G}(S) ;|\Re|=m \text { and } O_{S}(\Re)=1\right\} \text {. }
$$

Take an element $\mathfrak{S}$ of $\mathscr{C} \mathscr{C}(G)$. And let:

and

$$
\mathfrak{S}=\left\{H_{k} ; 1 \leqq k \leqq m\right\} ;
$$

$$
\mathfrak{S}_{i}=\left\{H_{k} ; 1 \leqq k \neq i \leqq m\right\}, 1 \leqq i \leqq m
$$


In this section, we shall investigate the relations among the elements of $\mathfrak{B}$, and also the relations between the elements of $\mathfrak{F}$ and the other elements of $\widetilde{\mho}_{G}(S)$.

We shall employ the above notation throughout this paper.

Lemma 5.1. Let $\Re$ be a subfamily of $\widetilde{\mho}_{G}(S)$. Then

(a) whenever $|\mathfrak{H}| \leqq 2, O_{S}(\Re) \supset 1$; in particular,

(b) $m \geqq 3$.

Proof. Take a subfamily $\Re$ of $\mathfrak{\mho}_{\theta}(S)$ with $|\Re| \leqq 2$. Then, Lemma $3.11(\mathrm{~h})$ implies that $\Omega_{1} Z(S), \Omega_{1} Z \hat{J}(S)$ or $J_{e}(S)$ is contained in $O_{S}(\Re)$, which proves this lemma.

Lemma 5.2. Fix $k=1,2, \cdots, m$. Then

(a) $H_{k}$ is neither 2-closed, nor 2'-closed;

(b) $1 \subset O_{S}\left(H_{k}\right) \subset S$;

(c) for some prime $p_{k} \geqq 5, H_{k}$ is a $\left\{2, p_{k}\right\}$-group with a nonidentity Sylow $p_{k}$-subgroup.

Proof. Suppose that $H_{k}$ is 2-closed or 2 -closed. Let $T=O_{S}\left(\mathfrak{S}_{k}\right)$. Since $\left|\mathfrak{F}_{k}\right|<m, T \supset 1$. Obviously, $H_{k}=N_{H_{k}}(T) O\left(H_{k}\right)$ and $H_{i}=$ $N_{H_{i}}(T) O\left(H_{i}\right)$ for all $i ; 1 \leqq i \neq k \leqq m$. Therefore, $1 \subset T \leqq O_{S}(\mathfrak{S})$, which is a contradiction. Hence, (a) is proved.

By Lemma 5.1, $1 \subset O_{S}\left(H_{k}\right)$, and by (a) and Lemma 4.2, $O_{S}\left(H_{k}\right) \subset$ $S$, which proves (b).

By Lemma $3.11(\mathrm{~b}), H_{k}$ is a $\left\{2, p_{k}\right\}$-group with a nonidentity Sylow $p_{k}$-subgroup for some odd prime $p_{k}$. Then by (a) and Lemma 3.9, $p_{k} \neq 3$, which proves (c).

The next lemma follows from Lemma 5.2(a) and 4.3(b):

LeMmA 5.3. Fix $k=1,2, \cdots, m$. Then

(a) $f_{\mathfrak{5}_{k}}\left(H_{i}\right) \geqq 1$ for all $i ; 1 \leqq i \neq k \leqq m$; and

(b) $f_{\mathfrak{F}_{k}}\left(H_{j}\right)=1$ for some $j ; 1 \leqq j \neq k \leqq m$.

Since $O_{S}(\mathfrak{S})=1$ by definition, the next lemma follows from Lemma 4.4 .

Lemma 5.4. There does not exist a subgroup $T$ of $S$ with the following properties: 
(1) $1 \subset T \subset S$;

(2) $T \triangleleft N_{G}(S)$; and

(3) $\left[O^{2}\left(H_{i}\right), O_{S}\left(H_{i}\right)\right] \leqq T$ for all $i ; 1 \leqq i \leqq m$.

LEMMA 5.5. There does not exist $H_{k}$ such that

$$
\left[O^{2}\left(H_{k}\right), O_{S}\left(H_{k}\right)\right] \leqq O_{S}\left(H_{i}\right) \text { for all } i ; 1 \leqq i \leqq m
$$

Proof. Suppose that there exists an element $H_{k}$ of $\mathfrak{S}$ such that $\left[O^{2}\left(H_{k}\right), O_{S}\left(H_{k}\right)\right] \leqq O_{S}\left(H_{i}\right)$ for all $i ; 1 \leqq i \leqq m$. Let $H_{j}$ be an element of $\mathscr{S}_{k}$ such that $f_{\mathfrak{F}_{k}}\left(H_{j}\right)=\max .\left\{f_{\mathfrak{F}_{k}}\left(H_{i}\right) ; 1 \leqq i \neq k \leqq m\right\}$. Let $T=O_{s}\left(H_{j}\right)$. By Lemma 5.2(b),

$$
1 \subset T \subset S \text {, and } T \triangleleft N_{G}(S) \text {. }
$$

By Lemma 4.3(f),

$$
\left[O^{2}\left(H_{i}\right), O_{S}\left(H_{i}\right)\right] \leqq T \text { for all } i ; 1 \leqq i \neq k \leqq m .
$$

On the other hand, by the assumption,

$$
\left[O^{2}\left(H_{l_{k}}\right), O_{S}\left(H_{k}\right)\right] \subseteq T .
$$

By (5.5.2) and (5.5.3),

$$
\left[O^{2}\left(H_{i}\right), O_{S}\left(H_{i}\right)\right] \leqq T \text { for all } i ; 1 \leqq i \leqq m .
$$

Then, (5.5.1) and (5.5.4) contradict the preceding lemma. Hence this lemma is proved.

Definition. Define the mapping $\sigma$ from $\mathfrak{S}_{\mathcal{C}}$ to the family of all subsets of $\mathfrak{S}$ as follows: for any element $H_{k}$ of $\mathfrak{K}$,

$$
\sigma\left(H_{k}\right)=\left\{H_{i} \in \mathfrak{F}_{k} ; f_{\mathfrak{F}_{k}}\left(H_{i}\right)=1\right\} .
$$

We call $\sigma$ the eigen -mapping of $\mathfrak{S}$.

LEMMA 5.6. (a) $\sigma\left(H_{j}\right) \neq \varnothing$ for any element $H_{j}$ of $\mathfrak{S}$;

(b) $\sigma\left(H_{j}\right) \cap \sigma\left(H_{h}\right)=\varnothing$ for any distinct elements $H_{j}$ and $H_{h}$ of $\mathfrak{S}$; and

(c) $\sigma$ induces a permutation of $\mathfrak{F}$.

Proof. Since $\mathfrak{S}_{k} \neq \varnothing$ by Lemma 5.1, $1 \leqq k \leqq m$, (a) follows from Lemma 5.3(b). To prove (b), we assume that $\sigma\left(H_{j}\right) \cap \sigma\left(H_{h}\right) \neq \varnothing$ for some distinct elements $H_{j}$ and $E_{h}$ of $\mathfrak{S}_{\text {. }}$. Take $H_{k}$ in $\sigma\left(H_{j}\right) \cap \sigma\left(H_{h}\right)$. As $H_{k} \in \sigma\left(H_{j}\right)$, by Lemma 5.2(a) and Lemma 4.3(f),

$$
\left[O^{2}\left(H_{k}\right), O_{S}\left(H_{k}\right)\right] \leqq O_{S}\left(H_{i}\right) \text { for all } i ; 1 \leqq i \neq j \leqq m .
$$


Similarly, as $H_{k} \in \sigma\left(H_{h}\right)$,

$$
\left[O^{2}\left(H_{k}\right), O_{S}\left(H_{k}\right)\right] \leqq O_{S}\left(H_{i}\right) \text { for all } i ; 1 \leqq i \neq h \leqq m .
$$

By (5.6.1) and (5.6.2),

$$
\left[O^{2}\left(H_{k}\right), O_{S}\left(H_{k}\right)\right] \leqq O_{S}\left(H_{i}\right) \text { for all } i ; 1 \leqq i \leqq m,
$$

which contradicts the preceding lemma. Hence (b) is proved.

Since $|\mathfrak{S}|$ is finite, (c) follows from (a) and (b). Thus, this lemma is proved.

According to the preceding lemma, we may consider $\sigma$ as an element of a permutation group on $\mathfrak{S}$.

LEMMA 5.7. $\langle\sigma\rangle$ acts transitively on $\mathfrak{S}$.

Proof. Suppose that $\langle\sigma\rangle$ acts intransitively on $\mathfrak{F}$. Let $\Re$ be a $\langle\sigma\rangle$-orbit of $\mathfrak{S}$. Then $\Re \subset \mathfrak{F}$. So, $O_{\mathcal{S}}(\Re) \supset 1$. Let $H_{k}$ be an element of $\Omega$ such that $f_{\Omega}\left(H_{k}\right)=\min .\left\{f_{\Omega}(H) ; H \in \Omega\right\}$. By Lemma 4.3(f),

$$
\left[O^{2}\left(H_{k}\right), O_{S}\left(H_{k}\right)\right] \subseteq O_{S}\left(H_{i}\right) \text { for any element } H_{i} \text { of } \Re \text {. }
$$

Let $H_{j}$ be an element of $\mathfrak{S}$ such that $\sigma\left(H_{j}\right)=H_{k}$. Then

$$
H_{j} \text { lies in } \Re \text {. }
$$

As $f_{\mathfrak{y}_{j}}\left(H_{k}\right)=1$, Lemma $4.3(\mathfrak{f})$ shows that

$$
\left[O^{2}\left(H_{k}\right), O_{S}\left(H_{k}\right)\right] \subseteq O_{S}\left(H_{i}\right) \text { for all } i ; 1 \leqq i \neq j \leqq m .
$$

By (5.7.1), (5.7.2) and (5.7.3), $\left[O^{2}\left(H_{k}\right), O_{S}\left(H_{k}\right)\right] \subseteq O_{S}\left(H_{i}\right)$ for all $i ; 1 \leqq$ $i \leqq m$, which contradicts Lemma 5.5. Hence this lemma is proved.

By the preceding lemma, we may assume:

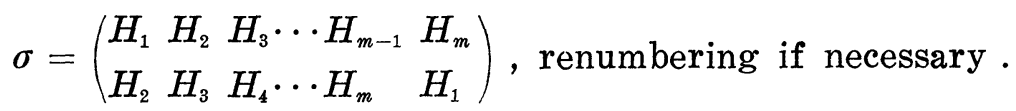

REMARK 5.2. In the following, without notice, all the suffixes of elements of $\mathfrak{S}$ will be used modulo $m$, if necessary. For instance, $H_{m+1}=H_{1}, H_{0}=H_{m}$, and so on.

LEMMA 5.8. Fix $k=1,2, \cdots, m$. Then

(a) $\left[O^{2}\left(H_{k}\right), O_{S}\left(H_{k}\right)\right] \subseteq O_{S}\left(H_{i}\right)$, if $i \not \equiv-1$ (modulo $m$ );

(b) $\left[O^{2}\left(H_{k}\right), O_{S}\left(H_{k}\right)\right] \nsubseteq O_{S}\left(H_{i}\right)$, if $i \equiv k-1$ (modulo $m$ ).

Proof. By definition, $f_{\mathfrak{p}_{k-1}}\left(H_{k}\right)=1$. So, by Lemma 5.3(a), $f_{\mathfrak{p}_{k-1}}\left(H_{k}\right)=\min .\left\{f_{\mathfrak{w}_{k-1}}\left(H_{i}\right) ; 1 \leqq i \neq k-1 \leqq m\right\}$. Then (a) follows from 
Lemma $4.3(\mathbf{f})$.

Suppose $\left[O^{2}\left(H_{k}\right), O_{S}\left(H_{k}\right)\right] \subseteq O_{S}\left(H_{k-1}\right)$. Then by (a), $\left[O^{2}\left(H_{k}\right), O_{S}\left(H_{k}\right)\right] \subseteq$ $O_{s}\left(H_{i}\right)$ for all $i ; 1 \leqq i \leqq m$, which contradicts Lemma 5.5. Hence (b) holds. This lemma is proved.

Lemma 5.9. Fix $k=1,2, \cdots, m$. Then

$$
1=f_{\mathfrak{⿰}_{k}}\left(H_{k+1}\right)<f_{\mathfrak{w}_{k}}\left(H_{k+2}\right)<\cdots<f_{\mathfrak{w}_{k}}\left(H_{m}\right)<f_{\mathfrak{w}_{k}}\left(H_{1}\right)<\cdots<f_{\mathfrak{w}_{k}}\left(H_{k-1}\right) .
$$

Proof. By the definition of $\sigma, f_{\mathfrak{w}_{k}}\left(H_{k+1}\right)=1$. Suppose $f_{\mathfrak{w}_{k}}\left(H_{i}\right) \geqq$ $f_{\mathfrak{w}_{k}}\left(H_{i}\right) \geqq f_{\mathfrak{F}_{k}}\left(H_{i+1}\right)$ for some $i ; 1 \leqq i \neq k-1, k \leqq m$. By Lemma 4.3(f), $\left[O^{2}\left(H_{i+1}\right), O_{S}\left(H_{i+1}\right)\right] \subseteq O_{S}\left(H_{i}\right)$, which contradicts Lemma 5.8(b). So, $f_{\mathfrak{w}_{k}}\left(H_{i}\right)<f_{\mathfrak{w}_{k}}\left(H_{i+1}\right)$ for all $i ; 1 \leqq i \neq k \leqq m$, which implies this lemma.

Lemma 5.10. Fix $k=1,2, \cdots, m$. Let $\left\{H_{i}, H_{i+1}, \cdots, H_{i+s}\right\}$ be a subset of $\mathfrak{\mathcal { B }}$. Let $\Omega$ be a subfamily of $\mathfrak{S}_{G}(S)$ which contains $\left\{H_{i}, H_{i+1}, \cdots, H_{i+s}\right\}$. Assume $O_{S}(\Re) \supset 1$. Then

(a) $f_{\Omega}\left(H_{i}\right)<f_{\Omega}\left(H_{i+1}\right)<\cdots<f_{\Omega}\left(H_{i+s}\right)$; and

(b) if $\Omega=\left\{H_{i}, H_{i+1}, \cdots, H_{i+s}\right\}$, then $f_{\mathfrak{\varepsilon}}\left(H_{i}\right)=1$.

Proof. Suppose $f_{\Omega}\left(H_{i+u}\right) \geqq f_{\Omega}\left(H_{i+u+1}\right)$ for some $u ; 0 \leqq u \leqq s-1$. Then by Lemma 4.3(f), $\left[O^{2}\left(H_{i+u+1}\right), O_{S}\left(H_{i+u+1}\right)\right] \leqq O_{S}\left(H_{i+u}\right)$, which contradicts Lemma 5.8(b). So, $f_{\Omega}\left(H_{i+u}\right)<f_{\Omega}\left(H_{i+u+1}\right)$ for all $u ; 0 \leqq$ $u \leqq s-1$, which implies (a).

(b) follows from Lemma 5.2(a) and Lemma 4.3(b).

Lemma 5.11. Fix $k=1,2, \cdots, m$. Suppose $M$ is a subgroup of $G$ which contains $N_{G}(S), H_{k}$ and $H_{k+1}$. Let $V$ be a normal subgroup of $M$. Then

(a) if $O^{2}\left(H_{k}\right) \nsubseteq V$, then $S \cap V \triangleleft H_{k}$ and $S \cap V \subseteq O_{S}\left(H_{k}\right)$;

(b) if $O^{2}\left(H_{k+1}\right) \nsubseteq V$, then $S \cap V \triangleleft H_{k+1}$ and $S \cap V \subseteq O_{S}\left(H_{k+1}\right)$;

(c) if $O^{2}\left(H_{k+1}\right) \subseteq V$, then $O^{2}\left(H_{k}\right) \subseteq V$;

(d) if $M=\left\langle N_{G}(S), H_{k}, H_{k+1}\right\rangle$ and $O^{2}\left(H_{k}\right) \nsubseteq V$, then $S \cap V \leqq$ $O_{S}\left(\left\{H_{k}, H_{k+1}\right\}\right)$ and $S \cap V \triangleleft M$.

Proof. (a) Since $O^{2}\left(H_{k}\right) \nsubseteq V$, by Lemma 5.2(a) and 3.11(e), $S \cap V \triangleleft H_{k}$. Since $S \cap V \triangleleft N_{G}(S)$, by Lemma 4.3(e), $S \cap V \cong$ $O_{S}\left(H_{k}\right)$, which proves (a). Similarly, we obtain (b).

Suppose $O^{2}\left(H_{k+1}\right) \subseteq V$ and $O^{2}\left(H_{k}\right) \nsubseteq V$. Then by (a), [O $O^{2}\left(H_{k+1}\right)$, $\left.O_{S}\left(H_{k+1}\right)\right] \subseteq V \cap S \subseteq O_{S}\left(H_{k}\right)$, which contradicts Lemma 5.8(b). Hence (c) is proved. (d) follows from (a), (b) and (c). Thus, this lemma is proved. 
REMARK 5.3. We note that all the statements from Lemma 5.1 to 5.11 are valid for any element of $\mathscr{C} \mathscr{L}(G)$. So, we can define the eigen-mapping for any element of $\mathscr{C} \mathscr{L}(G)$.

Lemma 5.12. Fix $k=1,2, \cdots, m$. Let $K$ be an element of $\Im_{G}(S)$, and let $\Re=\mathfrak{S}_{\mathfrak{I}} \cup\{K\}$. Assume that $\Re$ lies in $\mathscr{C} \mathscr{L}(G)$. Let $\tau$ be the eigen-mapping of $\Re$. Then

(a) $\tau\left(H_{i}\right)=H_{i+1}$, if $i \neq k-1, k$;

(b) $\tau(K)=H_{k+1}$; and

(c) $\tau\left(H_{k-1}\right)=K$.

Proof. Lemma 5.8 implies that the following $(\alpha),(\beta)$ and $(\gamma)$ are equivalent: for any element $L$ and $M$ of $\Omega$,

( $\alpha) \quad \tau(L)=M$

( $\beta) \quad\left[O^{2}(M), O_{S}(M)\right] \nsubseteq O_{S}(L)$; and

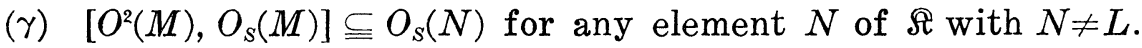
Therefore, (a) follows from the equivalence $(\alpha)$ to $(\beta)$. (b) follows from the equivalence $(\alpha)$ to $(\gamma)$. Then (c) follows from (a) and (b). By the preceding lemma and Lemma 5.8, we obtain:

Lemma 5.13. Fix $k=1,2, \cdots, m$. Let $K$ be an element of $\widetilde{F}_{G}(S)$. Assume that $\mathfrak{F}_{\mathfrak{s}} \cup\{K\}$ lies in $\mathscr{C} \mathscr{L}(G)$. Then

(a) $\left[O^{2}\left(H_{k+1}\right), O_{S}\left(H_{k+1}\right)\right] \nsubseteq O_{S}(K)$;

(b) $\left[O^{2}\left(H_{i}\right), O_{S}\left(H_{i}\right)\right] \leqq O_{S}(K)$ for all $i ; 1 \leqq i \neq k, k+1 \leqq m$;

(c) $\left[O^{2}(K), O_{S}(K)\right] \nsubseteq O_{S}\left(H_{k-1}\right)$; and

(d) $\left[O^{2}(K), O_{S}(K)\right] \subseteq O_{S}\left(H_{i}\right)$ for all $i ; 1 \leqq i \neq k-1, k \leqq m$.

Lemma 5.14. Fix $k=1,2, \cdots, m$. Suppose $\left\{K_{i} ; 1 \leqq i \leqq t\right\}$ is a subfamily of $\widetilde{\mho}_{G}(S)$. Let $T$ be a subgroup of $S$. Assume:

(1) $T \triangleleft N_{G}(S)$;

(2) $T \triangleleft K_{i}$ and $K_{i} / T$ is 2'-closed; and

(3) $\mathfrak{S}_{k} \cup\left\{K_{i}\right\}$ lies in $\mathscr{C} \mathscr{L}(G)$ for all $i ; 1 \leqq i \leqq t$. Then

$$
S=O_{S}\left(H_{k+1}\right)\left(\bigcap_{i=1}^{t} O_{S}\left(K_{i}\right)\right)
$$

Proof. Let $V=\bigcap_{i=1}^{t} O_{S}\left(K_{i}\right)$. By (1), (2) and Lemma 4.3(e), $T \subseteq V . \quad$ By $(2)$,

(5.14.1) $\left[O^{2}\left(K_{i}\right), O_{S}\left(K_{i}\right)\right] \subseteq T \subseteq V \subseteq V O_{S}\left(H_{k+1}\right)$ for all $i$; $1 \leqq i \leqq t$.

By (3) and Lemma 5.13(b), $\left[O^{2}\left(H_{i}\right), O_{S}\left(H_{i}\right)\right] \subseteq O_{S}\left(K_{i}\right)$ for all $i, j ; 1 \leqq$ $j \neq k-1, k \leqq m$ and $1 \leqq i \leqq t$. Therefore,

(5.14.2) $\left[O^{2}\left(H_{j}\right), O_{S}\left(H_{j}\right)\right] \subseteq V \subseteq V O_{S}\left(H_{k+1}\right)$ for all $j ; 1 \leqq j \neq k-1, k \leqq m$. 
on the other hand, obviously,

$$
\left[O^{2}\left(H_{k+1}\right), O_{S}\left(H_{k+1}\right)\right] \leqq O_{S}\left(H_{k+1}\right) \leqq V O_{S}\left(H_{k+1}\right) .
$$

Suppose $V O_{S}\left(H_{k+1}\right) \subset S$. Then by (5.14.1), (5.14.2), (5.14.3) and Lemma 4.4, $O_{S}\left(\mathfrak{S}_{k} \cup\left\{K_{i}\right\}\right) \supset 1$ for all $i ; 1 \leqq i \leqq t$, which contradicts (3). Hence $S=V O_{S}\left(H_{k+1}\right)$, as required.

Next, we shall prove the dual statement of the preceding lemma in some sense.

Lemma 5.15. Fix $k=1,2, \cdots, m$. Suppose $\Omega=\left\{K_{i} ; 1 \leqq i \leqq t\right\}$ is a subfamily of $\mathfrak{\Im}_{G}(S)$. Let $T$ be a subgroup of $S$. Assume:

(1) $T \triangleleft N_{G}(S)$;

(2) $K_{i} \triangleright T$, and $K_{i} / T$ is 2'-closed; and

(3) $O_{S}\left(\mathfrak{S}_{\Re} \cup\left\{K_{i}\right\}\right) \supset 1$ for all $i ; 1 \leqq i \leqq t$.

Then

$$
O_{S}\left(\mathscr{S}_{\Re} \cup \Re\right) \supset 1
$$

Proof. We shall use induction on $t$.

By (1), (2) and Lemma 4.3(e),

$$
T \subseteq O_{S}\left(K_{i}\right) \text { for all } i ; 1 \leqq i \leqq t .
$$

Let $\Omega_{i}=\mathscr{F}_{k} \cup\left\{K_{i}\right\}, 1 \leqq i \leqq t$. Assume that $O_{S}\left(K_{j}\right)=S$ for some $j ; 1 \leqq j \leqq t$. Let $V=O_{S}\left(\bigcup_{1 \leqq i \neq j \leqq t} \Re_{i}\right)$. By induction and definition,

$$
V \supset 1 \text { and } L=N_{L}(V) O(L) \text { for all } L \in \bigcup_{1 \leqq i \neq j \leqq t} \Re_{i} \text {. }
$$

On the other hand, by Lemma 4.2 ,

$$
K_{j}=N_{K j}(V) O\left(K_{j}\right) .
$$

By (5.15.2) and (5.15.3), $1 \subset V \subseteq O_{S}\left(\mathfrak{S}_{\Re} \cup \Re\right)$, as required. Hence, we may assume that:

$$
1 \subset O_{S}\left(K_{i}\right) \subset S \text { for all } i ; 1 \leqq i \leqq t .
$$

Next, assume that: for some $j ; 1 \leqq j \leqq t, f_{\AA_{j}}\left(K_{j}\right)=\max .\left\{f_{\AA_{j}}(L) ; L \in\right.$ $\Re_{j}$. Then by Lemma 4.3(f),

$$
\left[O^{2}\left(H_{i}\right), O_{S}\left(H_{i}\right)\right] \subseteq O_{S}\left(K_{j}\right) \text { for all } i ; 1 \leqq i \neq k \leqq m .
$$

By (2) and (5.15.1),

$$
\left[O^{2}\left(K_{i}\right), O_{S}\left(K_{i}\right)\right] \leqq T \leqq O_{S}\left(K_{j}\right) \text { for all } i ; 1 \leqq i \leqq t .
$$

By (5.15.4), (5.15.5), (5.15.6) and Lemma 4.4, $O_{S}\left(\mathscr{S}_{\mathfrak{C}} \cup \Re\right) \supset 1$, as required. Thus we may assume that: 


$$
f_{\mathfrak{s}_{i}}\left(K_{i}\right)<\max .\left\{f_{\AA_{i}}(L) ; L \in \Re_{i}\right\} \text { for all } i ; 1 \leqq i \leqq t .
$$

By Lemma 5.10(a),

$$
f_{\mathfrak{\varepsilon}_{i}}\left(H_{k-1}\right)=\max .\left\{f_{\aleph_{i}}(L) ; L \in \mathfrak{R}_{i}\right\} \text { for all } i ; 1 \leqq i \leqq t .
$$

By Lemma 4.3(f),

$$
\left[O^{2}\left(H_{j}\right), O_{S}\left(H_{j}\right)\right] \leqq O_{S}\left(H_{k-1}\right) \text { for all } j ; 1 \leqq j \neq k \leqq m
$$

and

$$
\left[O^{2}\left(K_{i}\right), O_{S}\left(K_{i}\right)\right] \leqq O_{S}\left(H_{k-1}\right) \text { for all } i ; 1 \leqq i \leqq t .
$$

Thus, by (5.15.7) and (5.15.8), Lemmas 4.4 and 5.2(b), $O_{S}\left(\mathscr{\mathcal { H }}_{k} \cup \Re\right) \supset 1$, which proves this lemma.

Definition. Let $\Re=\left\{K_{i} ; 1 \leqq i \leqq m\right\}$ be an element of $\mathscr{C} \mathscr{L}(G)$, and $\tau$ be the eigen-mapping of $\Omega$.

Then we may assume that: $\tau\left(K_{i}\right)=K_{i+1}$ for all $i ; 1 \leqq i \leqq m-1$, and $\tau\left(K_{m}\right)=K_{1}$, renumbering if necessary.

Let $\mathscr{Y}(\Re)=\left\{K \in \Re ; K \neq N_{K}\left(J_{e}(S)\right)\right\}$. Then $\mathscr{Y}(\Re) \neq \varnothing$; otherwise $1 \subset J_{e}(S) \cong O_{S}(\Re)$, a contradiction to the fact that $\Re$ lies in $\mathscr{C} \mathscr{L}(G)$.

So, we may assume that $K_{1}$ lies in $\mathscr{Y}(\Re)$, by (cyclically) renumbering, if necessary.

Let $\mathscr{V}(\Re)=\left\{K_{1}=K_{i_{1}}, K_{i_{2}}, \cdots, K_{i_{\lambda}}\right\}$, where $1=i_{1}<i_{2}<\cdots<i_{\lambda}$. For each element $K_{i_{\nu}}$ of $\mathscr{Y}(\Re), 1 \leqq \nu \leqq \lambda$, we define:

$$
\text { if }|\mathscr{Y}(\Re)| \geqq 2, \alpha\left(\Re: K_{i_{\nu}}\right)=\left\{K_{t} ; i_{\nu} \leqq t<i_{\nu+1}\right\}, 1 \leqq \nu \leqq \lambda ;
$$

and

$$
\text { if } \mathscr{Y}(\Re)=\left\{K_{1}\right\}, \alpha\left(\Re: K_{1}\right)=\left\{K_{t} ; 1 \leqq t \leqq m-1\right\} .
$$

Define $\beta\left(\Re: K_{i_{\nu}}\right)=\left\{K \in \alpha\left(\Omega: L_{i_{\nu}}\right) ;\left[O^{2}(K), \Omega_{1} Z \hat{J}\left(O_{S}\left(\alpha\left(\Re: K_{i_{\nu}}\right)\right) J_{e}(S)\right)\right]=1\right\}$. Finally, we define $\delta(\Re)=\sum_{\nu=1}^{\lambda}\left|\beta\left(\Omega: K_{i_{\nu}}\right)\right|$.

REMARK 5.4 (i). We can easily verify that for any element $\Re$ of $\mathscr{C} \mathscr{L}(G)$ and any element $K$ of $\mathscr{Y}(\Re), \alpha(\Omega: K), \beta(\Re: K)$ and $\delta(\Re)$ are defined independently of numbering of the elements of $\Omega$.

(ii) By definition, if $|\mathscr{Y}(\Re)| \geqq 2$, then $\Re=\bigcup_{\nu=1}^{\lambda} \alpha\left(\Re: K_{i \nu}\right)$ (disjoint union).

Lemma 5.16. Suppose $H_{j}$ is an element of $\mathscr{Y}(\mathfrak{S})$. Let $\Re=$ $\alpha\left(\Re: H_{j}\right), T=O_{S}(\Re) J_{e}(S)$, and $A=\left\langle N_{G}(S), H \in \Re\right\rangle$. Then
(a) $\left[\Omega_{1} Z(S), O^{2}\left(H_{j}\right)\right]=\left[\Omega_{1} Z \widehat{J}(T), O^{2}\left(H_{j}\right)\right]=1$;
(b) $\Omega_{1} Z \hat{J}(T) \triangleleft A$; and
(c) $\left[\Omega_{1} Z(S), H_{j-1}\right]=1$. 
Proof. By Lemma 3.2,

(5.16.1) $\Omega_{1} Z(S) \leqq \Omega_{1} Z \widehat{J}(S) \subseteq \Omega_{1} Z \widehat{J}(T)$, and $J_{e}(T)=J_{e}(S)$.

Since $\mathfrak{R} \subset \mathscr{S}_{j-1}$, by Lemma 5.10(b), $f_{\Re}\left(H_{j}\right)=1$. By Lemma 4.3(c), $T$ is a Sylow 2-subgroup of $O^{2}\left(H_{j}\right) T$. Let $L=O^{2}\left(H_{j}\right) T$. By Theorem 3.1(b), $L=N_{L}\left(J_{e}(T)\right) C_{L}\left(\Omega_{1} Z \hat{J}(T)\right) O(L)$. So, by (5.16.1),

$$
H_{j}=N_{I I j}\left(J_{e}(S)\right)\left(C_{H_{j}}\left(\Omega_{1} Z \hat{J}(T)\right) S\right)(O(L) S) .
$$

Since $H_{j} \neq N_{H_{j}}\left(J_{e}(S)\right)$ and $H_{j} \neq O\left(H_{j}\right) S$,

$$
H_{j}=C_{H_{j}}\left(\Omega_{1} Z \hat{J}(T)\right) S .
$$

(a) follows from (5.16.1) and (5.16.2).

To prove (b), take any element $H_{k}$ of $\Re$ with $H_{k} \neq H_{j}$, if possible. Since $H_{k}=N_{H_{k}}\left(J_{e}(S)\right), H_{k}=N_{H_{k}}(T)$. So,

$$
T \triangleleft H_{k} \text {. }
$$

Therefore, (b) follows from (a). To prove (c), by Lemma 3.11(h), we may assume that:

$$
H_{j-1}=N_{H_{j-1}}(\hat{J}(S)) .
$$

Let $B=\left\langle N_{G}(S), H_{j-1}, H_{j}\right\rangle$, and $B_{0}=C_{B}\left(\Omega_{1} Z \hat{J}(S)\right)$. By (a) and (5.16.4), $O^{2}\left(H_{j}\right) \subseteq B_{0} \triangleleft B$. Then by Lemma 5.11(c), $O^{2}\left(H_{j-1}\right) \subseteq B_{0}$, so that $\left[\Omega_{1} Z(S), O^{2}\left(H_{j-1}\right)\right] \leqq\left[\Omega_{1} Z \widehat{J}(S), O^{2}\left(H_{j-1}\right)\right]=1$, which proves (c), and this lemma.

Lemma 5.17. Let $H_{j}$ be an element of :/(占). Assume:

(1) $H_{k+1} \in \beta\left(\mathfrak{S}_{\mathfrak{g}}: H_{j}\right)$; and

(2) $H_{k} \in \alpha\left(\mathfrak{S}: H_{j}\right)$,

then

$$
H_{k} \in \beta\left(\mathfrak{S}_{\mathcal{E}}: H_{j}\right) .
$$

Proof. Let $T=O_{S}\left(\alpha\left(\mathscr{H}_{\mathcal{g}}: H_{j}\right)\right) J_{e}(S), B=\left\langle N_{G}(S), H_{k}, H_{k+1}\right\rangle$, and $B_{0}=C_{B}\left(\Omega_{1} Z \hat{J}(T)\right)$. By Lemma 5.16(b), $B_{0} \triangleleft B$. By (1), $O^{2}\left(H_{k+1}\right) \subseteq B_{0}$. Then by Lemma 5.11(c), $O^{2}\left(H_{k}\right) \subseteq B_{0}$, which shows that $H_{k} \in \beta\left(\mathfrak{S}_{:}: H_{j}\right)$, as required. Hence this lemma is proved.

LeMma 5.18. There exists a trio of elements $H_{j}, H_{k}$ and $H_{k+1}$ of $\mathfrak{S}$ which satisfies the following conditions:

(a) $H_{j} \in \mathscr{Y}(\mathfrak{S})$;

(b) $H_{k} \in \beta\left(\mathfrak{S}_{\mathrm{S}}: H_{j}\right)$; and

(c) $H_{k+1} \in \alpha\left(\mathfrak{S}_{\mathfrak{E}}: H_{j}\right) \backslash \beta\left(\mathfrak{S}_{\mathfrak{C}}: H_{j}\right)$.

(Here, it is admissible also that $H_{j}=H_{k}$.) 
Proof. Suppose that for any element $H_{j}$ of $\mathscr{Y}(\mathfrak{E}), \beta\left(\mathfrak{S}_{\mathfrak{Z}}: H_{j}\right)=$ $\alpha\left(\mathfrak{S}: H_{j}\right)$. Assume $|\mathscr{Y}(\mathscr{S})| \geqq 2$. Then, from the definitions, we have that $\mathfrak{S}=\bigcup_{H_{j}} \beta\left(\mathfrak{S}_{\mathfrak{S}}: H_{j}\right)$ (disjoint union), where $H_{j}$ ranges over all the elements of $\mathscr{Y}(\mathfrak{S})$. Hence by definition, $H_{i}=C_{H_{i}}\left(\Omega_{1} Z(S)\right)$ for all $i ; 1 \leqq i \leqq m$, so that $1 \subset \Omega_{1} Z(S) \leqq O_{S}(\mathfrak{S})$, a contradiction. Next, assume $|\mathscr{Y}(\mathfrak{S})|=1$. Let $\mathscr{Y}(\mathfrak{S})=\left\{H_{j}\right\}$. By definition and the assumption, $\quad \mathfrak{S}=\alpha\left(\mathfrak{K}: H_{j}\right) \cup\left\{H_{j-1}\right\}=\beta\left(\mathfrak{S}_{\mathfrak{C}}: H_{j}\right) \cup\left\{H_{j-1}\right\} . \quad$ By Lemma 5.16(c) and definition, $1 \subset \Omega_{1} Z(S) \subseteq O_{S}(\mathscr{S})$, a contradiction. Therefore, there exists an element $H_{j}$ of $\mathscr{Y}(\mathfrak{K})$ such that $\beta\left(\mathfrak{S}_{\mathfrak{L}}: H_{j}\right) \subset \alpha\left(\mathfrak{S}_{\mathfrak{E}}: H_{j}\right)$. Let $\alpha\left(\mathfrak{Q}: H_{j}\right)=\left\{H_{j}, H_{j+1}, \cdots, H_{j+t}\right\}$. By Lemma 5.17, we may assume that $\beta\left(S_{\mathfrak{S}:} H_{j}\right)=\left\{H_{j} H_{j+1}, \cdots, H_{j+s}\right\}$, where $s<t$. Setting $H_{k}=H_{j+s}$, we have this lemma.

Lemma 5.19. Suppose that $H_{j}, H_{k}$, and $H_{k+1}$ are elements of $\mathfrak{F}_{\text {, }}$ and $L$ is an element of $\mathfrak{\mho}_{G}(S)$. Let $\Re=\mathfrak{S}_{k} \cup\{L\}$. Assume:

(1) $H_{j} \in \mathscr{Y}(\mathfrak{Q})$;

(2) $H_{k} \in \beta\left(\mathfrak{S}_{\mathcal{E}}: H_{j}\right)$;

(3) $H_{k+1} \in \alpha\left(\mathfrak{S}_{2}: H_{j}\right) \backslash \beta\left(\mathfrak{S}_{\mathfrak{C}}: H_{j}\right)$;

(4) $\Omega$ lies in $\mathscr{C} \mathscr{L}(G)$;

(5) $\left\langle N_{G}(S), L, H_{k+1}\right\rangle \leqq\left\langle N_{G}(S), H_{k}, H_{k+1}\right\rangle$; and

(6) $L=N_{L}\left(J_{e}(S)\right)$ if and only if $H_{k}=N_{H_{k}}\left(J_{e}(S)\right)$.

Then

(a) Assume $L \neq N_{L}\left(J_{e}(S)\right)$. Then

(a.1) $H_{k+1} \in \alpha(\Omega: L)$;

(a.2) $\delta(\Re) \geqq \delta(\mathfrak{E})$; and

(a.3) if $H_{k+1} \in \beta(\Re: L), \delta(\Re)>\delta(\mathfrak{S})$.

(b) Assume $L=N_{L}\left(J_{e}(S)\right)$ and $\left[O^{2}(L), \Omega_{1} Z \widehat{J}\left(O_{S}\left(\alpha\left(\mathfrak{S}_{\varepsilon}: H_{j}\right)\right) J_{e}(S)\right)\right]=1$. Then

(b.1) $H_{j} \in \mathscr{Y}(\Re)$ and $H_{k+1} \in \alpha\left(\Omega: H_{j}\right)$;

(b.2) $\delta(\Re) \geqq \delta(\mathfrak{S})$; and

(b.3) if $H_{k+1} \in \beta\left(\Re: H_{j}\right), \delta(\Re)>\delta(\mathfrak{E})$.

Proof. In this proof, we shall quote the results of Lemma 5.12 without notice.

Let $\mathscr{Y}(\mathfrak{E})=\left\{H_{j}=H_{j_{1}}, H_{j_{2}}, \cdots, H_{j_{t}}\right\}$.

To prove (a), assume $L \neq N_{L}\left(J_{e}(S)\right)$. By (1), (2) and (6), $H_{j}=$ $H_{k}$. So, by (3) and Lemma 5.17,

$$
\beta\left(\mathfrak{S}_{\mathrm{E}}: H_{j}\right)=\left\{H_{j}\right\} .
$$

Then it is easily verified that

$$
\begin{gathered}
\mathscr{Y}(\Re)=\left\{L, H_{j_{2}}, H_{j_{3}}, \cdots, H_{j_{t}}\right\}, \alpha(\Re: L)=\{L\} \cup \alpha\left(\mathfrak{S}: H_{j}\right) \backslash\left\{H_{j}\right\}, \\
\text { and whenever } 2 \leqq i \leqq t, \beta\left(\Re: H_{j_{i}}\right)=\beta\left(S_{\mathcal{E}}: H_{j_{i}}\right) .
\end{gathered}
$$


Then (a.1) follows from this fact.

By (5.19.1) and (5.19.2),

$$
\begin{aligned}
\delta(\Re) & =\sum_{i=2}^{t}\left|\beta\left(\Re: H_{j_{i}}\right)\right|+|\beta(\Re: L)| \\
& \geqq \sum_{i=2}^{t}\left|\beta\left(\Re: H_{j_{i}}\right)\right|+1 \\
& =\sum_{i=2}^{t}\left|\beta\left(\mathfrak{S}: H_{j_{i}}\right)\right|+\left|\beta\left(\mathfrak{S}: H_{j}\right)\right| \\
& =\delta(\mathfrak{S}),
\end{aligned}
$$

which proves (a.2). Then we observe that equality holds in the above if and only if $\beta(\Omega: L)=\{L\}$, which proves (a.3).

Next, assume $L=N_{L}\left(J_{e}(S)\right)$. By (1), (2), and (6), $H_{k} \neq H_{j}$. Then, it is easily verified that:

$$
\begin{gathered}
\mathscr{Y}(\Re)=\mathscr{Y}(\mathfrak{S}) \text {, and whenever } 2 \leqq i \leqq t, \beta\left(\Re: H_{j_{i}}\right) \\
=\beta\left(\mathfrak{E}: H_{j_{i}}\right) \text {; moreover, }
\end{gathered}
$$$$
\alpha\left(\Re: H_{j}\right)=\{L\} \cup \alpha\left(\mathfrak{S}_{\mathcal{C}}: H_{j}\right) \backslash\left\{H_{k}\right\} \text {. }
$$

Then, (b.1) follows from (5.19.3) and (5.19.4). By (5) and (5.19.4), $\left\langle N_{G}(S), K \in \alpha\left(\Omega: H_{j}\right)\right\rangle \subseteq\left\langle N_{G}(S), K \in \alpha\left(\mathfrak{S}_{\mathcal{E}}: H_{j}\right)\right\rangle . \quad$ By Lemma 4.3(a), $O_{S}\left(\alpha\left(\Re: H_{j}\right)\right) J_{e}(S) \supseteqq O_{S}\left(\alpha\left(\mathfrak{S}_{2}: H_{j}\right)\right) J_{e}(S)$. Thus, by Lemma 3.2(c),

$$
\Omega_{1} Z \widehat{J}\left(O_{S}\left(\alpha\left(\Re: H_{j}\right)\right) J_{e}(S)\right) \cong \Omega_{1} Z \widehat{J}\left(\left(\alpha\left(\mathscr{S}_{E}: H_{j}\right)\right) J_{e}(S)\right) .
$$

By (5.19.4) and (5.19.5),

$$
\beta\left(\mathfrak{S}_{\mathrm{C}}: H_{j}\right) \backslash\left\{H_{k}\right\} \cong \beta\left(\Re: H_{j}\right) .
$$

Since $L \in \beta\left(\Re: H_{j}\right)$ by the assumption of (b) and (5.19.5),

$$
\left|\beta\left(\Omega: H_{j}\right)\right| \geqq\left|\beta\left(\mathscr{S}_{\mathrm{g}}: H_{j}\right)\right| \text {. }
$$

By (5.19.3) and (5.19.7),

$$
\begin{aligned}
\delta(\mathfrak{S}) & =\sum_{i=2}^{t}\left|\beta\left(\mathfrak{S}: H_{j_{i}}\right)\right|+\left|\beta\left(\mathfrak{S}: H_{j}\right)\right| \\
& \leqq \sum_{i=2}^{t}\left|\beta\left(\Re: H_{j_{i}}\right)\right|+\left|\beta\left(\Re: H_{j}\right)\right| \\
& =\delta(\Re),
\end{aligned}
$$

which proves (b.2).

By (5.19.6), equality holds in the above if and only if $\beta\left(\Omega: H_{j}\right)=$ $\{L\} \cup \beta\left(\mathfrak{S}_{\mathrm{E}}: H_{j}\right) \backslash\left\{H_{k}\right\}$. Then (3) yields (b.3). Hence this lemma is proved.

Lemma 5.20. Suppose that $H_{j}, H_{k}$ and $H_{k+1}$ are elements of $\mathfrak{S}$, 
and $L$ is an element of $\widetilde{F}_{G}(S)$. Let $\Re=\mathfrak{F}_{k+1} \cup\{L\}$. Assume:

(1) $H_{j} \in \mathscr{Y}(\mathfrak{S})$;

(2) $H_{k} \in \beta\left(\mathfrak{S}_{\mathcal{E}}: H_{j}\right)$;

(3) $H_{k+1} \in \alpha\left(\mathfrak{S}: H_{j}\right) \backslash \beta\left(\mathfrak{S}_{c}: H_{j}\right)$;

(4) $\Re$ lies in $\mathscr{C} \mathscr{L}(G)$;

(5) $\left\langle N_{G}(S), H_{k}, L\right\rangle \leqq\left\langle N_{G}(S), H_{k}, H_{k+1}\right\rangle$; and

(6) $\left[O^{2}(L), \Omega_{1} Z \widehat{J}\left(O_{S}\left(\alpha\left(\mathfrak{S}: H_{j}\right)\right) J_{e}(S)\right)\right]=1$.

Then

$$
\delta(\Re)>\delta(\mathfrak{S})
$$

Proof. Let $: \mathscr{V}\left(\mathfrak{S}_{\mathrm{C}}\right)=\left\{H_{j}=H_{j_{1}}, H_{j_{2}}, \cdots, H_{j_{1}}\right\}$.

It is easily verified that

$$
\text { whenever } 2 \leqq i \leqq t, \beta\left(\Re: H_{j_{i}}\right)=\beta\left(\mathfrak{S}_{\mathrm{c}}: H_{j_{i}}\right) \text {. }
$$

First, assume $L \neq N_{L}\left(J_{e}(S)\right)$. Then

$$
\mathscr{Y}(\mathfrak{K})=\left\{L, H_{j_{1}}, H_{j_{2}}, \cdots, H_{j_{t}}\right\} .
$$

By (2), (3) and Lemma 5.17,

$$
\beta\left(\Re: H_{j}\right)=\beta\left(\mathfrak{F}: H_{j}\right) .
$$

By (5.20.1), (5.20.2) and (5.20.3),

$$
\begin{aligned}
\delta(\Re) & =\sum_{i=2}^{t}\left|\beta\left(\Re: H_{j_{i}}\right)\right|+\left|\beta\left(\Re: H_{j}\right)\right|+|\beta(\Re: L)| \\
& >\sum_{i=2}^{t}\left|\beta\left(\mathfrak{S}: H_{j_{i}}\right)\right|+\left|\beta\left(\mathfrak{S}: H_{j}\right)\right| \\
& =\delta(\mathfrak{S}) .
\end{aligned}
$$

Next, assume $L=N_{L}\left(J_{c}(S)\right)$. Then it is easily verified that

$$
\mathscr{Y}(\Re)=\mathscr{U}(\mathfrak{C}) \text {; and }
$$

$$
\alpha\left(\Re: H_{j}\right)=\alpha\left(\mathfrak{S}: H_{j}\right) \cup\{L\} \backslash\left\{H_{k+1}\right\} .
$$

By (5) and (5.20.5),

$$
\left\langle N_{G}(S), K \in \alpha\left(\Re: H_{j}\right)\right\rangle \subseteq\left\langle N_{\alpha}(S), K \in \alpha\left(\mathfrak{S}_{2}: H_{j}\right)\right\rangle .
$$

Then by Lemma $4.3(\mathrm{a})$ and $3.2(\mathrm{c})$,

(5.20.6) $\quad \Omega_{1} Z \hat{J}\left(O_{S}\left(\alpha\left(\Omega: H_{j}\right)\right) J_{e}(S)\right) \subseteq \Omega_{1} Z \hat{J}\left(O_{S}\left(\alpha\left(S_{\mathcal{C}}: H_{j}\right)\right) J_{e}(S)\right)$.

$\operatorname{By~(3),~(6)~and~(5.20.6),~} L \in \beta\left(\Re: H_{j}\right)$ and $\beta\left(\Re: H_{j}\right) \supset \beta\left(\mathscr{S}_{\varepsilon}: H_{j}\right)$, so, $\left|\beta\left(\Omega: H_{j}\right)\right|>\left|\beta\left(\mathfrak{K}: H_{j}\right)\right|$.

By (5.20.1) and (5.20.7), $\delta(\Re)>\delta(\mathfrak{S})$, which completes the proof of 
this lemma.

6. On some groups involved in the minimal situation.

Notation. Fix a trio of elements $H_{j}, H_{k}$ and $H_{k+1}$ of $\mathfrak{S}$ which satisfies the conclusions (a), (b) and (c) of Lemma 5.18.

Let

$$
\begin{aligned}
& M=\left\langle N_{G}(S), H_{k}, H_{k+1}\right\rangle ; \\
& N=\left\langle N_{G}(S), H_{k+1}\right\rangle ; \\
& W=\left\langle O^{2}\left(H_{k}\right)^{M}\right\rangle ;
\end{aligned}
$$

and

$$
S_{0}=W \cap O_{S}\left(\left\{H_{k}, H_{k+1}\right\}\right) .
$$

By Lemma 5.2(c), we recall that for some odd prime $q \geqq 5, H_{k}$ is a $\{2, q\}$-group with a nonidentity Sylow $q$-subgroup. From now on, $q$ denotes the prime such that $H_{k}$ is a $\{2, q\}$-group.

LEMMA 6.1. (a) $W=\left\langle O^{2}\left(H_{k}\right)^{N}\right\rangle \triangleleft M$;

(b) $\left[W, \Omega_{1} Z \widehat{J}\left(O_{S}\left(\alpha\left(\mathscr{S}_{c}: H_{j}\right)\right) J_{e}(S)\right)\right]=1$;

(c) $\left[O^{2}\left(H_{k+1}\right), \Omega_{1} Z \hat{J}\left(O_{S}\left(\alpha\left(\mathscr{S}_{E}: H_{j}\right)\right) J_{e}(S)\right)\right] \neq 1$, in particular

$$
O^{2}\left(H_{k+1}\right) \nsubseteq W \text {. }
$$

Proof. $W=\left\langle O^{2}\left(H_{k}\right)^{N}\right\rangle \triangleleft\left\langle O^{2}\left(H_{k}\right), N\right\rangle=M$, which proves (a). (b) follows from (a) and Lemma 5.16(a).

Since the trio of elements $H_{j}, H_{k}$ and $H_{k+1}$ satisfies the conclusions of Lemma 5.18, the former part of (c) follows from Lemma 5.18(c). Then the latter part of (c) follows from (b).

LEMma 6.2. (a) $H_{k} / S_{0}$ is $q$-closed;

(b) $1 \subset S_{0} \triangleleft M$, in particular $S_{0} \triangleleft N_{G}(S)$;

(c) $1 \subset O_{S}\left(H_{k+1}\right)=S \cap O_{2}(N) \triangleleft N$;

(d) for any $S$-invariant subnormal subgroup $V$ of $M$, $S$ is normal in some Sylow 2-subgroup of VS.

Proof. Let $\Re=\left\{H_{k}, H_{k+1}\right\}$. By Lemma 5.1(a), $1 \subset O_{S}(\Re)$. Then by Lemma $5.10(\mathrm{~b}), f_{\AA}\left(H_{k}\right)=1$. Hy Lemma 4.3(c),

$H_{k} / W(\Omega: 1)$ is $q$-closed.

Since $O^{2}\left(H_{k}\right) \subseteq W$ and $W(\Re: 1)=O_{S}(\Re)$,

$$
\left[O^{2}\left(H_{k}\right), O_{S}(\Re)\right] \subseteq W \cap O_{S}(\Re)=S_{0} .
$$


Then, (a) follows from (6.2.1) and (6.2.2).

Suppose $S_{0}=1$. Then by (a), $H_{k}$ is $q$-closed, which contradicts Lemma 5.2(a). Hence, we obtain that $1 \subset S_{0}$. Since $O_{S}(\Re) \triangleleft M$, by Lemma 6.1(a),

$$
S_{0}=W \cap O_{S}(\Re) \triangleleft M,
$$

which proves the former part of (b).

Since $N_{G}(S) \subseteq M$, the latter part of (b) follows from (6.2.3), By Lemma 5.2(b), $1 \subset O_{S}\left(H_{k+1}\right)$. Since $N=\left\langle N_{G}(S), H_{k+1}\right\rangle$, by Lemma 4.3(a), $N \triangleright O_{S}\left(H_{k+1}\right)=S \cap O_{2}(N)$, which shows (c). Since $1 \subset S_{0} \triangleleft$ $N_{G}(S)$ by (b), the assumption (1) of the theorem implies that $S$ is normal in some Sylow 2-subgroup $U$ of $N_{G}\left(S_{0}\right)$. Since $U \subseteq N_{G}(S) \subseteq$ $M \cong N_{G}\left(S_{0}\right), U$ is a Sylow 2-subgroup of $M$. Since $V$ is subnormal in $M$, by Lemma 3.3(a), $V \cap U$ is a Sylow 2-subgroup of $V$. So, $S(V \cap U)$ is a Sylow 2-subgroup of $V S$. Since $S(V \cap U) \subseteq U$, (d) is proved.

Notation. Let $K$ be the pre-image of $O\left(W / S_{0}\right)$ in $W$. And by Lemma $6.2(\mathrm{~d})$, let $U$ be a Sylow 2-subgroup of $M$ in which $S$ is normal.

LeMma 6.3. Let $\bar{X}=X K / K$ for any subgroup $X$ of $M$. Then

(a) $O^{2}\left(\bar{H}_{k}\right)=O\left(\bar{H}_{k}\right)=O_{q}\left(\bar{H}_{k}\right)$;

(b) $O_{2}(\bar{W}) \cap \bar{S}=1$;

(c) $O_{2}(\bar{W})=Z(\bar{W})=F_{\infty}(\bar{W}) \subseteq Z\left(\bar{W} \bar{H}_{k+1}\right)$.

Proof. (a) follows from Lemma 6.2(a). To prove (b) and (c), we may assume that $\bar{W} \neq 1$, so that

$$
O^{2}\left(\bar{H}_{k}\right) \nsubseteq O_{2}(\bar{W}) \text {. }
$$

Let $S_{1}$ be the intersection of $S$ and the pre-image of $O_{2}(\bar{W})$ in $M$. Then by (6.3.1) and Lemma 5.11(d), $S_{1} \subseteq O_{S}\left(\left\{H_{k}, H_{k+1}\right\}\right) \cap W=S_{0}$. So, $O_{2}(\bar{W}) \cap \bar{S}=\bar{S}_{1} \subseteq \bar{S}_{0}=1$, which proves $(\mathrm{b})$.

Since $O_{2}(\bar{W}) \triangleleft \bar{U}$ and $\bar{S} \triangleleft \bar{U}$, by $(\mathrm{b}),\left[O_{2}(\bar{W}), \bar{S}\right] \cong O_{2}(\bar{W}) \cap \bar{S}=1$. Thus, $\bar{S} \subseteq C_{\bar{M}}\left(O_{2}(\bar{W})\right) \triangleleft \bar{M}$. Then by Lemma 3.11(f),

$$
\bar{H}_{k}, \bar{H}_{k+1} \subseteq C_{\bar{u}}\left(O_{2}(\bar{W})\right) \text {. }
$$

Since $O(\bar{W})=1$, by (6.3.2),

$$
F_{\infty}(\bar{W}) \leqq O_{2}(\bar{W}) \cong Z\left(\bar{W} \bar{H}_{k+1}\right) .
$$

On the other hand, generally,

(6.3.4) $Z(\bar{W}) \subseteq O_{2}(\bar{W}) \subseteq F_{\infty}(\bar{W})$, and $\bar{W} \cap Z\left(\bar{W} \bar{H}_{k+1}\right) \subseteq Z(\bar{W})$. 
Then, !(c) follows from (6.3.3) and (6.3.4). Hence, this lemma is proved.

Definition. Let 2 be the family of all the subgroups $L$ of $M$ that satisfy the following conditions:

( $\alpha) \quad L \in \mathfrak{\mho}_{G}(S)$;

(B) $L$ is a $\{2, q\}$-group;

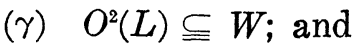

(ס) $L / S_{0}$ is $q$-closed.

Let

$$
\stackrel{尺}{1}_{1}=\left\{L \in \mathbb{R} ; O_{S}\left(\mathfrak{S}_{k} \cup\{L\}\right) \supset 1\right\} ;
$$

and

$$
\stackrel{\Omega}{2}_{2}=\left\{L \in \mathbb{R} ; O_{S}\left(\mathfrak{H}_{k} \cup\{L\}\right)=1\right\} \text {. }
$$

And let

$$
Y=N_{M}\left(O_{S}\left(\mathfrak{S}_{k} \cup \Omega_{1}\right)\right) O(W)
$$

LeMma 6.4. (a) $H_{k}$ lies in $\Omega_{2}$;

(b) for any element $L$ of $\mathfrak{S}_{2}, \mathfrak{F}_{k} \cup\{L\}$ lies in $\mathscr{C} \mathscr{L}(G)$;

(c) $O_{S}\left(\mathfrak{S}_{k} \cup \Omega_{1}\right) \supset 1$.

Proof. Obviously, $H_{k}$ is a $\{2, q\}$-group which lies in $\widetilde{\mho}_{G}(S)$. By definition, $O^{2}\left(H_{k}\right) \subseteq W$. By Lemma $6.2(\mathrm{a}), H_{k} / S_{0}$ is $q$-closed. Hence, $H_{k}$ lies in $\stackrel{2}{2}$. Suppose $H_{k}$ lies in $\mathfrak{\Omega}_{1}$. Then by definition, $1 \subset O_{S}\left(\mathfrak{S}_{k} \cup\right.$ $\left.\left\{H_{k}\right\}\right)=O_{s}(\mathfrak{E})$, which is a contradiction. Hence, (a) is proved.

(b) follows from the definitions.

(c) follows from Lemma 5.15.

Lemma 6.5. Suppose $D$ is a normal subgroup of $M$. Assume:

(1) $K \subseteq D \subseteq W$; and

(2) $S_{0} \subset D \cap S$.

Then

$$
D=W
$$

Proof. Suppose $D \subset W$. Since $D \triangleleft M$, it must be that $O^{2}\left(H_{k}\right) \nsubseteq$ D. Then by Lemma 5.11(d), $D \cap S \subseteq O_{S}\left(\left\{H_{k}, H_{k+1}\right\}\right) \cap W=S_{0}$, which contradicts (2). Hence, this lemma is proved.

Assumption (A). In the following discussion, without loss of generality, we may assume that:

$$
\delta(\mathfrak{S})=\max .\{\delta(\Re) ; \Re \in \mathscr{C} \mathscr{L}(G)\} ;
$$


and for any element $L$ of $\mathcal{R}_{2}$,

$\left\langle O^{2}(L)^{x} ; x \in\left\langle N_{G}(S), H_{k+1}\right\rangle\right\rangle=W$, if $L$ satisfies the following conditions:

( $\alpha) \quad \delta\left(\mathscr{S}_{k} \cup\{L\}\right)=\delta\left(\mathfrak{S}_{\mathcal{E}}\right)$; and

( $\beta$ ) the trio of elements $H_{j}, L$ and $H_{l+1}$ satisfies the conclusions (a), (b), and (c) of Lemma 5.18 with $H_{k}$ replaced by $L$.

LEMMA 6.6. Let $V$ be a normal subgroup of $M$ such that $S_{0} \leqq$ $V \subseteq K$, and let $D_{1}, D_{2}, \cdots, D_{n}$ be $S$-invariant normal subgroups of $W$ all of which contain $V$. Assume:

(1) $O^{2}\left(H_{k}\right) \cong D_{1} D_{2} \cdots D_{n}$.

Let $\bar{X}=X V / V$ for any subgroup $X$ of $M$.

(2) $\bar{D}_{i} \cap\left\langle\bar{D}_{j} ; 1 \leqq j \neq i \leqq n\right\rangle$ is a 2-group for all $i$; $1 \leqq i \leqq n$;

(3) $\left[\bar{D}_{i}, \bar{D}_{j}\right]=1$ for all $i, j: 1 \leqq i \neq j \leqq n$.

Then there exists an element $L$ of $\mathbb{S}_{2}$ which satisfies the following conditions:

(a) for some $i$; $1 \leqq i \leqq n, O^{2}(L) \subseteq D_{i}$;

(b) $\left\langle O^{2}(L)^{x} ; x \in N\right\rangle=W$;

(c) $\mathscr{S}_{k} \cup\{L\}$ lies in $\mathscr{C} \mathscr{L}(G)$;

(d) $\delta\left(\mathfrak{S}_{k} \cup\{L\}\right)=\delta(\mathfrak{T})$; and

(e) replacing $H_{k}$ by $L$ and $\mathfrak{S}_{\mathfrak{C}}$ by $\mathscr{S}_{k} \cup\{L\}$, the conclusions of Lemma 5.18(a), (b) and (c) are satisfied.

Proof. By Lemma 6.2(d), $S$ is normal in some Sylow 2-subgroup of $\left(D_{1} D_{2} \cdots D_{n}\right) S$. Since $S_{0} \leqq V \leqq K, V$ is 2-closed, and $O_{2}(V)=S_{0}=$ $S \cap V$. Hence, all the assumptions of Proposition 3.13 are satisfied, for $q=r,\left(D_{1} D_{2} \cdots D_{n}\right) S=D, H_{k}=H$, and the other notation as is.

So, there exist $S$-irreducible subgroups $\left\{L_{i} ; 1 \leqq i \leqq t\right\}$ of $\left(D_{1} D_{2} \cdots D_{n}\right) S$ which satisfy all the conclusions of Proposition 3.13.

Let $F=\left\langle L_{i} ; 1 \leqq i \leqq t\right\rangle$. By Lemma 3.13(a) and (b),

$F$ is a solvable $\{2, q\}$-group with a Sylow 2-subgroup

$$
S \text {, and } H_{k} \subseteq F \text {. }
$$

Since $\left[O\left(H_{k}\right), O_{2}(F)\right]=1=\left[O\left(L_{i}\right), O_{2}(F)\right]$, (6.6.1) implies that:

$$
O\left(H_{k}\right) \subseteq O(F) \text { and } O\left(L_{i}\right) \leqq O(F) \text { for all } i ; 1 \leqq i \leqq t
$$

Since $H_{k} / S_{0}$ is $q$-closed by Lemma $6.2($ a), by Proposition $3.13(\mathrm{~d})$ and (g), $\quad F V / V$ is $q$-closed, so that $F / S_{0}$ is $q$-closed. And $O^{2}(F) \subseteq$ $D_{1} D_{2} \cdots D_{n} \leqq W$. Hence, $L_{i}$ lies in $\&$ for all $i ; 1 \leqq i \leqq t$.

Suppose $L_{i}$ lies in $\mathbb{Q}_{1}$ for all $i ; 1 \leqq i \leqq t$. Then by (6.6.2) and 
Lemma 6.4(c), $F=N_{F}\left(O_{S}\left(\mathscr{S}_{k} \cup \mathfrak{R}_{1}\right)\right)$ and $O_{S}\left(\mathfrak{\mathcal { F }}_{k} \cup \mathfrak{\Omega}_{1}\right) \supset 1$. Since $H_{k} \cong$ $F$ by (6.6.1), $H_{k}=N_{H_{k}}\left(O_{S}\left(\mathscr{S}_{k} \cup \mathfrak{\Omega}_{1}\right)\right) O\left(H_{k}\right)$, which shows that $1 \subset$ $O_{S}\left(\mathfrak{S}_{k} \cup \mathfrak{R}_{1}\right) \subseteq O_{S}(\mathfrak{S})$. This is a contradiction. Hence, we may assume that $L_{1}$ lies in $\Omega_{2}$.

Let $L=L_{1}$ and $\Re=\mathfrak{S}_{k} \cup\{L\}$. By Lemma 6.4(b),

$\Re$ lies in $\mathscr{C} \mathscr{L}(G)$,

which proves (c).

By Proposition 3.13(d),

$L$ lies in $D_{i}$ for some $i ; 1 \leqq i \leqq n$,

which proves (a). By (6.6.3) and Assumption (A.1),

$$
\delta(\mathfrak{S}) \geqq \delta(\Re) .
$$

On the other hand, by Proposition 3.13(e) and (f),

$$
H_{k}=N_{H_{k}}\left(J_{e}(S)\right) \text { if and only if } L=N_{L}\left(J_{e}(S)\right) \text {. }
$$

Then all the assumptions of Lemma 5.19 are satisfied. Assume $H_{k} \neq N_{H_{k}}\left(J_{e}(S)\right)$. Then by (6.6.6), $L \neq N_{L}\left(J_{e}(S)\right)$. By (6.6.5) and Lemma 5.19(a),

$$
\delta(\mathfrak{\mathcal { Z }})=\delta(\Re), L \in \mathscr{Y}(\Re) \text { and } H_{k+1} \in \alpha(\Re: L) \backslash \beta(\Re: L) .
$$

Next, assume $H_{k}=N_{H_{k}}\left(J_{e}(S)\right)$. Then by (6.6.6), $L=N_{L}\left(J_{e}(S)\right)$. Since $O^{2}(L) \subseteq W$, by Lemma 6.1(b), $\left[O^{2}(L), \Omega_{1} Z \hat{J}\left(O_{S}\left(\alpha\left(\mathscr{S}_{\varepsilon}: H_{j}\right)\right) J_{e}(S)\right)\right]=$ 1. Then by (6.6.5) and Lemma $5.19(\mathrm{~b})$,

$$
\delta(\Re)=\delta(\mathfrak{S}), H_{j} \in \mathscr{Y}(\Re) \text { and } H_{k+1} \in \alpha\left(\Omega: H_{j}\right) \backslash \beta\left(\Re: H_{j}\right) \text {. }
$$

Hence, we obtain (d) and (e) by (6.6.7) and (6.6.8). Then, (b) follows from Assumption (A.2). Hence this lemma is proved.

LEMMA 6.7. Suppose $D_{1}$ is a normal subgroup of $M$ such that $K \subseteq D_{1} \subseteq W . \quad$ Then

$$
D_{1} / K \subseteq Z(W / K) \text { or } D_{1}=W .
$$

Proof. Let $D_{2}=C_{M}\left(D_{1} / K\right) \cap W$. Then, $K \subseteq D_{2} \triangleleft M$. Suppose $O^{2}\left(H_{l}\right) \subseteq D_{1} D_{2}$. Then, $D_{1} D_{2}=W$. In this proof, let $\bar{X}=X K / K$ for any subgroup $X$ of $M$. By Lemma 6.3(c), $\bar{D}_{1} \cap \bar{D}_{2} \subseteq Z(\bar{W})=O_{2}(\bar{W})$, and $\left[\bar{D}_{1}, \bar{D}_{2}\right]=1$. Then by Lemma $6.6(\mathrm{a})$ and (b), for some $i ; 1 \leqq$ $i \leqq 2, D_{i}=\left\langle D_{i}^{x} ; x \in M\right\rangle=W$, which implies that $D_{1}=W$ or $\bar{D}_{1}=$ $Z(\bar{W})$, as required. Hence, we may assume that $O^{2}\left(H_{k}\right) \nsubseteq D_{1} D_{2}$. Since $O^{2}\left(H_{k}\right) \subseteq W, O^{2}\left(H_{k}\right) \nsubseteq D_{1} C_{M}\left(D_{1} / K\right)$.

Then by Lemma 5.11(a), 


$$
S \cap D_{1} C_{M}\left(D_{1} / K\right) \subseteq O_{S}\left(H_{k}\right) .
$$

On the other hand, by Lemma 6.5, we may assume that

$$
\bar{D}_{1} \cap \bar{S} \subseteq \bar{S}_{0}=1 \text {. }
$$

By Lemma 3.3(a),

$$
\bar{U} \cap \bar{D}_{1} \text { is a Sylow 2-subgroup of } \bar{D}_{1} .
$$

By (6.7.2),

$$
\left[\bar{U} \cap \bar{D}_{1}, \bar{S}\right] \subseteq \bar{D}_{1} \cap \bar{S}=1 .
$$

Let $\bar{F}=\bar{D}_{1} \bar{H}_{k+1}$ and $\bar{C}=\left\langle\bar{S}^{x} ; x \in N_{\bar{F}}\left(\bar{U} \cap \bar{D}_{1}\right)\right\rangle$. Then, by (6.7.4),

$$
\left[\bar{U} \cap \bar{D}_{1}, \bar{C}\right]=1 \text {. }
$$

Since $O\left(\bar{D}_{1}\right) \subseteq O(\bar{W})=1$ by Lemma $6.3(\mathrm{c}),(6.7 .5)$ and a theorem of G. Glauberman [2] imply that

$$
\bar{C} / C_{\bar{o}}\left(\bar{D}_{1}\right) \text { has a normal 2-complement. }
$$

On the other hand, since $O^{2}\left(\bar{H}_{k}\right)=\bar{H}_{k}$ by Lemma 5.2(a) and 3.11(f), the Frattini argument and the isomorphism theorem imply that:

(6.7.7) $\bar{C} /\left(\bar{C} \cap \bar{D}_{1} C_{\bar{F}}\left(\bar{D}_{1}\right)\right) \simeq \bar{F} / \bar{D}_{1} C_{\bar{F}}\left(\bar{D}_{1}\right) \simeq \bar{H}_{k+1} /\left(\bar{H}_{k+1} \cap \bar{D}_{1} C_{\bar{F}}\left(\bar{D}_{1}\right)\right)$.

By (6.7.6) and (6.7.7), $\bar{H}_{k+1} /\left(\bar{H}_{k+1} \cap \bar{D}_{1} C_{\bar{F}}\left(\bar{D}_{1}\right)\right)$ has a normal 2-complement. So, $H_{k+1} /\left(H_{k+1} \cap D_{1} C_{M}\left(D_{1} / K\right)\right)$ has a normal 2-complement. It follows that

$$
\left[O^{2}\left(H_{k+1}\right), O_{S}\left(H_{k+1}\right)\right] \subseteq S \cap D_{1} C_{M}\left(D_{1} / K\right) .
$$

By (6.7.1) and (6.7.8), $\left[O^{2}\left(H_{k+1}\right), O_{S}\left(H_{k+1}\right)\right] \subseteq O_{S}\left(H_{k}\right)$, which contradicts Lemma 5.8(b). Hence this lemma is proved.

LeMma 6.8. $O^{2}\left(C_{W}\left(S_{0}\right)\right)=O(W)$.

Proof. Obviously, $O^{2}\left({ }_{W}\left(S_{0}\right)\right) \supseteq O(W)$. To prove the opposite inclusion, first, we assume that $S_{0} C_{W}\left(S_{0}\right) \subseteq F_{\infty}(W)$. Then by Lemma 6.3(c), $S_{0} C_{W}\left(S_{0}\right) / S_{0}$ is $2^{\prime}$-closed. It follows that $S_{0} C_{W}\left(S_{0}\right)$ has a normal 2-complement. Since $S_{0} C_{W}\left(S_{0}\right) \triangleleft W, O^{2}\left(C_{W}\left(S_{0}\right)\right) \subseteq O(W)$, as required. Hence, we may assume that

$$
S_{0} C_{W}\left(S_{0}\right) \nsubseteq F_{\infty}(W) .
$$

By Lemma 6.3(c) and the preceding lemma, we have that $K C_{W}\left(S_{0}\right)=$ $W$. For $D_{1}=C_{W}\left(S_{0}\right) S_{0}, D_{2}=K$ and $V=D_{1} \cap D_{2}$, all the assumptions of Lemma 6.6 are satisfied. By Lemma 6.6(a) and (b), for some $i ; 1 \leqq i \leqq 2, D_{i}=\left\langle D_{i}^{x} ; x \in N\right\rangle=W$, that is, $K=W$ or $S_{0} C_{W}\left(S_{0}\right)=W$. 
Suppose $K=W$. Then, $S_{0} C_{W}\left(S_{0}\right) \subseteq K \subseteq F_{\infty}(W)$, which contradicts (6.8.1). Thus, we have that $S_{0} C_{W}\left(S_{0}\right)=W$. It follows that $\left[O^{2}\left(H_{k}\right)\right.$, $\left.S_{0}\right] \subseteq\left[O^{2}(W), S_{0}\right]=1$. Then by Lemma 6.2(a), $H_{k}$ is $2^{\prime}$-closed, which contradicts Lemma 5.2(a). Hence this lemma is proved.

Lemma 6.9. For any element $L$ of $\stackrel{\mathcal{Q}}{\sim}, L$ lies in $\mathfrak{\Omega}_{1}$ if and only if $L \subseteq Y$, in particular $H_{k} \nsubseteq Y$.

Proof. Suppose $L$ lies in $\Omega_{1}$. Then, $L=N_{L}\left(O_{S}\left(\mathfrak{S}_{k} \cup \mathbb{\Omega}_{1}\right)\right) O(L)$. Since $O(L) \subseteq O^{2}\left(C_{W}\left(S_{0}\right)\right)$, by Lemma 6.8, $O(L) \cong O(W)$. Hence, $L \cong$ $N_{M}\left(O_{S}\left(\mathfrak{S}_{k} \cup \Omega_{1}\right)\right) O(W)=Y$, as required.

Conversely, suppose $L \subseteq Y$. Then, $L=N_{L}\left(O_{S}\left(\mathfrak{F}_{k} \cup \mathbb{R}_{1}\right)\right) O(L)$. Hence by Lemma 6.4(c), $1 \subset O_{S}\left(\mathfrak{S}_{k} \cup \Re_{1}\right) \subseteq O_{S}\left(\mathfrak{F}_{k} \cup\{L\}\right)$, which shows that $L$ lies in $\Omega_{1}$. Since $H_{k}$ lies in $\Omega_{2}$ by Lemma 6.4(a), we have the latter part of this lemma. Hence this lemma is proved.

LEMMA 6.10. Suppose $V$ is a normal subgroup of $M$ such that $S_{0} \subseteq V \subseteq F_{\infty}(W)$. Let $\bar{X}=X V / V$ for any subgroup $X$ of $M$, and $\bar{T}=\bigcap_{L \in \mathfrak{\Omega}_{2}} C_{\bar{S}}\left(O_{q}(\bar{L})\right)$. Then

(a) $\bar{S}=\overline{O_{S}\left(H_{k+1}\right)} \bar{T}$;

(b) $O^{2}(\bar{L})=O_{q}(\bar{L})$ for any element $L$ of $\&$. For any $\bar{S}$-invariant q-subgroup $\bar{R}$ of $\bar{W}$,

(c) there exist elements $\left\{L_{i} ; 1 \leqq i \leqq t\right\}$ of \& such that $\bar{R}=$ $\left\langle O_{q}\left(\bar{L}_{i}\right) ; 1 \leqq i \leqq t\right\rangle ;$ and

(d) $[\overline{\bar{R}}, \bar{T}] \leqq \bar{Y}$.

Proof. For any element $L$ of $\&, L / S_{0}$ is $q$-closed by definition, so that

$$
L / V \text { is } q \text {-closed, that is, } O^{2}(\bar{L})=O_{q}(\bar{L}),
$$

which proves (b).

Let $T_{0}=\bigcap_{L \in \varepsilon_{2}} O_{S}(L)$. Then by Lemma 5.14 and 6.4(b),

$$
S=O_{S}\left(H_{k+1}\right) T_{0} .
$$

By (6.10.1), if $L \in \mathfrak{\Omega}_{2},\left[O_{q}(\bar{L}), \bar{T}_{0}\right] \subseteq O_{q}(\bar{L}) \cap \bar{T}_{0}=1$, which shows that $\bar{T}_{0} \subseteq \bar{T}$. Then, (a) follows from (6.10.2).

Let $D^{*}$ be a Hall $\{2, q\}$-subgroup of the pre-image of $\bar{S} \bar{R}$ in $M$ which contains $S$. Then,

$$
O_{q}\left(\bar{D}^{*}\right)=\bar{R}
$$

By Lemma 6.3(c), $D^{*} / S_{0}$ is $q$-closed. 
Let $D$ be the pre-image of $O_{q}\left(D^{*} / S_{0}\right)\left(S / S_{0}\right)$ in $M$. By (6.10.3) and (6.10.4),

$$
O_{q}(\bar{D})=\bar{R}
$$

Since $S$ is a Sylow 2-subgroup of $D$, by Lemma 3.10, there exist $S$-irreducible subgroups $\left\{L_{i} ; 1 \leqq i \leqq t\right\}$ of $D$ with a Sylow 2-subgroup $S$, and $D=\left\langle L_{i} ; 1 \leqq i \leqq t\right\rangle$. Then, it is easily verified that $L_{i}$ lies in $\&$ for all $i ; 1 \leqq i \leqq t$. Hence, (c) is proved. To prove (d), we may assume that $\bar{R}=[\bar{R}, \bar{T}]$. Then, by (a), $O_{q}\left(\bar{L}_{i}\right) \leqq \Phi(\bar{R})$ for all $L_{i} \in \mathbb{R}_{2}$. By the preceding lemma, $\bar{R}=\left\langle O_{q}\left(\bar{L}_{i}\right), \Phi(\bar{R}) ; L_{i} \in \mathbb{\Omega}_{1}\right\rangle=$ $\langle\bar{R} \cap \bar{Y}, \Phi(\bar{R})\rangle=\bar{R} \cap \bar{Y}$, which proves (d).

LEMMA 6.11. (a) $N_{G}(S) \subseteq N \subseteq Y$;

(b) $Y$ contains a Hall $\{2, q\}$-subgroup of KS which contains $S$.

Proof. Since $O_{s}\left(\mathfrak{S}_{\mathfrak{C}_{k}} \cup \mathbb{\Omega}_{1}\right) \triangleleft\left\langle N_{G}(S), H_{k+1}\right\rangle=N$,

$$
\mathrm{S} \subseteq N_{G}(S) \subseteq N \leqq N_{M}\left(O_{S}\left(\mathscr{S}_{k} \cup \mathfrak{R}_{1}\right)\right) O(W)=Y,
$$

which proves (a).

Since $S$ is a Sylow 2-subgroup of $K S$, by (a), we need only show that $Y$ contains a Sylow $q$-subgroup of $K$. Suppose that $K$ does not contain any Sylow $q$-subgroup of $K$. By the main theorem of W. Feit and J. G. Thompson [1], $K$ possesses a chief factor $V_{1}$ l $V_{2}$ which satisfies the following conditions: $S_{0}$

$(\alpha)$ both $V_{1}$ and $V_{2}$ are normal subgroups of $M$ which contains

( $\beta$ ) $\quad V_{1} / V_{2}$ is a nonidentity elementary Abelian $q$-group; and

( $\gamma) Y$ contains a Sylow $q$-subgroup of $V_{2}$, but does not contain any Sylow $q$-subgroup of $V_{1}$.

Let $\bar{X}=X V_{2} / V_{2}$ for any subgroup $X$ of $M$. By $(\beta)$,

$$
\bar{V}_{1} \cap \bar{Y} \subset \bar{V}_{1} \text {. }
$$

By Lemmas 6.10(b), (c) and 6.9,

there exist elements $\left\{L_{i} ; 1 \leqq i \leqq t\right\}$ of $\mathbb{S}_{2}$ such that

$$
\begin{gathered}
\bar{V}_{1}=\left(\bar{V}_{1} \cap \bar{Y}\right)\left\langle O_{q}\left(\bar{L}_{i}\right) ; 1 \leqq i \leqq t\right\rangle \text { and } \\
O_{q}\left(\bar{L}_{2}\right) \nsubseteq \bar{V}_{1} \cap \bar{Y} \text { for all } i ; 1 \leqq i \leqq t .
\end{gathered}
$$

By Lemmas 3.11(g), 3.4, and 4.3(e),

$$
C_{S}\left(V_{1} / V_{1} \cap Y\right) \leqq O_{S}\left(L_{i}\right) \text { for all } i ; 1 \leqq i \leqq t .
$$

Since $\mathfrak{F}_{k} \cup\left\{L_{i}\right\}$ lies in $\mathscr{C} \mathscr{L}(G), 1 \leqq i \leqq t$, by Lemma 5.13(a),

$$
\left[O^{2}\left(H_{k+1}\right), O_{S}\left(H_{k+1}\right)\right] \nsubseteq O_{S}\left(L_{i}\right) \text { for all } i ; 1 \leqq i \leqq t .
$$


On the other hand, by Lemma 6.10(a),

$$
S=O_{S}\left(H_{k+1}\right) C_{S}\left(V_{1} / V_{1} \cap Y\right) \text {. }
$$

Since $H_{k+1} \subseteq Y$ by (a), $H_{k+1}$ leaves invariant $V_{1}$ and $V_{1} \cap Y$. So,

$$
C_{H_{k+1}}\left(V_{1} / V_{1} \cap Y\right) \triangleleft H_{k+1} .
$$

By (6.11.6) and (6.11.7), $S \subseteq O_{S}\left(H_{k+1}\right) C_{H_{k+1}}\left(V_{1} / V_{1} \cap Y\right) \triangleleft H_{k+1} . \quad$ By (6.11.7) and Lemma 3.11(f),

$$
O^{2}\left(H_{k+1}\right) \subseteq C_{H_{k+1}}\left(V_{1} / V_{1} \cap Y\right) \text {. }
$$

By (6.11.4) and (6.11.8), $\left[O^{2}\left(H_{k+1}\right), O_{S}\left(H_{k+1}\right)\right] \subseteq C_{S}\left(V_{1} / V_{1} \cap Y\right) \subseteq O_{S}\left(L_{i}\right)$ for all $i ; 1 \leqq i \leqq t$, which contradicts (6.11.5). Hence this lemma is proved.

Lemma 6.12. Let $\bar{X}=X K / K$ for any subgroup $X$ of $M$. Then

(a) $Z(\bar{W}) \subseteq \bar{Y}$

(b) $O_{q}\left(\bar{H}_{k}\right) \nsubseteq \bar{Y} \cap \bar{W} \subset \bar{W}=E(\bar{W})$; and

(c) $\mathscr{D}\left(\bar{W} \bar{H}_{k+1}: \pi\right)=\varnothing$.

Proof. By Lemmas 6.3(c) and 6.11(a), $Z(\bar{W})=O_{2}(\bar{W}) \leqq \bar{U} \leqq \overline{N_{G}(S)} \leqq$ $\bar{N} \subseteq \bar{Y}$, which proves (a).

Suppose $O_{q}\left(\bar{H}_{k}\right) \subseteq \bar{Y}$. Since $S \subseteq Y$ by Lemma $6.11(\mathrm{a}), \bar{H}_{k} \subseteq \bar{Y}$. Let $V$ be the pre-image of $\bar{H}_{k}$ in $M$, and let $D_{1}$ be a Hall $\{2, q\}$ subgroup of $V$ which contains $H_{k}$. By Lemma 6.11(b), $Y \cap V$ contains a Hall $\{2, q\}$-subgroup $D_{2}$ of $V$ which contains $S$. Since $S$ is a Sylow 2-subgroup of $V, S$ is a Sylow 2-subgroup of $D_{1}$ and $D_{2}$. By Lemma 3.5(b), $N_{V}(S)$ possesses an element $x$ such that $D_{2}^{x}=$ $D_{1}$. Since $x \in N_{V}(S) \subseteq Y$ by Lemma 6.11(a), $H_{k} \subseteq D_{1}=D_{2}^{x} \subseteq Y$, which contradicts Lemma 6.9. Hence, we obtain that $O_{q}\left(\bar{H}_{k}\right) \nsubseteq \bar{Y}$. Since $O_{q}\left(\bar{H}_{k}\right) \subseteq \bar{W}$, it follows that

$$
O_{q}\left(\bar{H}_{k}\right) \nsubseteq \bar{Y} \cap \bar{W} \subset \bar{W} .
$$

Hence, by (a), (6.12.1) and Lemma 6.3(c), $Z(\bar{W})=F_{\infty}(\bar{W}) \subset \bar{W}$. So, $E(\bar{W}) \neq 1$. Since $E(\bar{W}) \nsubseteq Z(\bar{W})$, by Lemma 6.7 ,

$$
E(\bar{W})=\bar{W} .
$$

Then, (b) follows from (6.12.1) and (6.12.2). Since $H_{k+1}$ is solvable, by Lemma 6.8 ,

$$
C_{W H_{k+1}}\left(S_{0}\right) \text { is solvable. }
$$

By (6.12.3) and the assumption (2) of the theorem, $\mathscr{D}\left(\bar{W} \bar{H}_{k+1}: \pi\right)=\mathscr{D}\left(W H_{k+1} / C_{W H_{k+1}}\left(S_{0}\right): \pi\right) \subseteq \mathscr{D}\left(N_{G}\left(S_{0}\right) / C_{G}\left(S_{0}\right): \pi\right)=\varnothing$, 
which proves (c). Hence this lemma is proved.

LEMMA 6.13. Let $K^{*}$ be the pre-image of $Z(W / K)$ in $W$, and $\bar{X}=X K^{*} / K^{*}$ for any subgroup $X$ of $M$. Then

(a) for some natural number $r$,

$\bar{W}=E(\bar{W})=\bar{E}_{1} \times \bar{E}_{2} \times \cdots \times \bar{E}_{r}$, where $\bar{E}_{k}$ is a non-Abelian

simple group with $\bar{E}_{k} \simeq \bar{E}_{1}$ for all $k ; 1 \leqq k \leqq r$;

(b) $\bar{E}_{k} \nsubseteq \bar{Y} \cap \bar{W}$ for all $k ; 1 \leqq k \leqq r$;

(c) $\bar{N}=\left\langle\bar{H}_{k+1}, N_{\bar{N}}(\bar{S})\right\rangle$;

(d) there exists a subgroup $\bar{T}$ of $\bar{S}$ such that

(d.1) $\bar{S}=\left(\bar{S} \cap O_{2}(\bar{N})\right) \bar{T}$, and

(d.2) $[\bar{R}, \bar{T}] \subseteq \bar{Y} \cap \bar{W}$ for any $\bar{S}$-invariant $q$-subgroup $\bar{R}$ of $\bar{W}$;

(e) renumbering of $\{1,2, \cdots, r\}$, if necessary, there exists an S-invariant q-subgroup $\bar{Q}$ of $\bar{W}$ such that $\left\langle\pi_{1}(\bar{Q})^{x} ; x \in \bar{N}\right\rangle=\bar{W}$, where $\pi_{1}$ denotes the projection mapping from $\bar{W}$ to $\bar{E}_{1}$.

Proof. By Lemma 6.12(b), for some natural number $r$,

$$
\bar{W}=E(\bar{W})=\bar{E}_{1} \times \bar{E}_{2} \times \cdots \times \bar{E}_{r} \text {, where } \bar{E}_{k} \text { is a }
$$
non-Abelian simple group, $1 \leqq k \leqq r$.

Let $\bar{W}_{0}=\left\langle\bar{E}_{1}^{x} ; x \in \bar{N}\right\rangle$. Since $\bar{M}=\langle\bar{W}, \bar{N}\rangle, \bar{W}_{0} \triangleleft \bar{M}$. Since $\bar{W}_{0} \nsubseteq$ $Z(\bar{W})$, by Lemma $6.7, \bar{W}_{0}=\bar{W}$, so that

$\bar{N}$ acts transitively on $\left\{\bar{E}_{k} ; 1 \leqq k \leqq r\right\}$.

Hence,

$$
\bar{E}_{k} \simeq \bar{E}_{1} \text { for all } k ; 1 \leqq k \leqq r .
$$

Thus, (a) follows from (6.13.1) and (6.13.3).

Suppose $\bar{E}_{k} \leqq \bar{Y}$ for some $k ; 1 \leqq k \leqq r$. Since $\bar{Y}$ is $\bar{N}$-invariant by Lemma $6.11(\mathrm{a}),(6.13 .2)$ shows that $\bar{W}=\left\langle\bar{E}_{k}^{x} ; x \in \bar{N}\right\rangle \subseteq \bar{Y}$, which contradicts Lemma $6.12(\mathrm{a})$ and (b). Hence, $\bar{E}_{k} \nsubseteq \bar{Y}$ for all $k ; 1 \leqq$ $k \leqq r$, which proves $(b)$.

Since $\bar{N}=\left\langle\bar{H}_{k+1}, \overline{N_{G}(S)}\right), \bar{N}=\left\langle\bar{H}_{k+1}, N_{\bar{N}}(\bar{S})\right\rangle$, which proves $($ c).

By Lemma 6.2(c),

$$
\overline{O_{S}\left(H_{k+1}\right)}=\bar{S} \cap \overline{O_{2}(N)} \subseteq \bar{S} \cap O_{2}(\bar{N}) .
$$

Let $\bar{T}=\bigcap_{L \in \Omega_{2}} C_{\bar{S}}\left(O_{q}(\bar{L})\right)$. Then by Lemma 6.10 and (6.13.4), $\bar{S}=$ $\overline{O_{S}\left(H_{k+1}\right)} \bar{T} \cong\left(\bar{S} \cap O_{2}(\bar{N})\right) \bar{T}$, and $[\bar{R}, \bar{T}] \leqq \bar{Y}$ for any $\bar{S}$-invariant $q$ subgroup $\bar{R}$ of $\bar{W}$, which proves (d).

By Lemma 3.6, $\bar{S}$ induces a permutation of $\Lambda=\{1,2, \cdots, r\}$ 
where we identity $k$ with $E_{k}, 1 \leqq k \leqq r$. Let $A_{1}, A_{2}, \cdots, A_{n}$ be the set of all $\bar{S}$-orbits of $\Lambda, \bar{D}_{i}=\left\langle\bar{E}_{\lambda} ; \lambda \in \Lambda_{i}\right\rangle$, and let $D_{i}$ be the preimage of $\bar{D}_{i}$ in $M, 1 \leqq i \leqq n$. Then, for $V=K$ and $D=W S$, all the assumptions of Lemma 6.6 are satisfied. By Lemma 6.6(a), (b) and Lemma $6.10(\mathrm{~b})$, there exists an element $L$ of $\mathfrak{R}_{2}$, such that

$$
\left\langle O_{q}(\bar{L})^{x} ; x \in \bar{N}\right\rangle=\bar{W} \text { and } O_{q}(\bar{L}) \leqq \bar{D}_{i} \text { for some } i ; 1 \leqq i \leqq n .
$$

Renumbering $\Lambda$, if necessary, we may assume that $\bar{D}_{i}=\left\langle\bar{E}_{1}^{x} ; x \in \bar{S}\right\rangle$. Since $\bar{L}$ is $\bar{S}$-irreducible by Lemma $3.11(a)$,

$$
\begin{aligned}
O_{q}(\bar{L}) \cong & \left\langle\pi_{1}\left(O_{q}(\bar{L})\right)^{x} ; x \in S\right\rangle, \text { where } \pi_{1} \text { denotes the projection } \\
& \text { mapping from } \bar{W} \text { to } \bar{E}_{1} .
\end{aligned}
$$

By (6.13.5) and (6.13.6), $\left\langle\pi_{1}\left(O_{q}(\bar{L})\right)^{x} ; x \in \bar{N}\right\rangle=\bar{W}$, which proves (e). Hence this lemma is proved.

LeMmA 6.14. $O^{2}\left(H_{k+1}\right) \subseteq W C_{M}(W / K)$.

Proof. Let $K^{*}$ be the pre-image of $Z(W / K)$ in $W$. Then by Lemma $6.12(\mathrm{c}), \quad \mathscr{D}\left(W H_{k+1} / K^{*}: \pi\right) \subseteq \mathscr{D}\left(W H_{k+1} / K: \pi\right)=\varnothing$. So, by Lemma 6.13 and Proposition 3.17, we obtain that $O^{2}\left(H_{k+1}\right) \subseteq$ $W C_{M}\left(W / K^{*}\right)$. Hence, it is enough to show that $C_{M}(W / K) \supseteqq C_{M}\left(W / K^{*}\right)$. Let $\bar{X}=X K / K$ for any subgroup $X$ of $M$. Then, $\bar{W}$ stabilizes a normal series: $\overline{C_{M}\left(W / K^{*}\right)}=C_{\bar{M}}(\bar{W} / Z(\bar{W})) \supseteqq Z(\bar{W}) \supseteqq 1$. By Lemma 6.12(b), $O^{2}(\bar{W})=\bar{W} r$, so that by Lemma $3.7\left[\bar{W}, \overline{C_{M}\left(W / K^{*}\right)}\right]=1$. Therefore, $C_{M}\left(W / K^{*}\right) \subseteq C_{M}(W / K)$, as required. Hence this lemma is proved.

Notation. By Lemma 5.2(c), $H_{k+1}$ is a $\{2, p\}$-group with a nonidentity Sylow $p$-subgroup. From now, $p$ denotes the prime such that $H_{k+1}$ is a $\{2, p\}$-group.

LEMMA 6.15. All the assumptions of Proposition 3.13 are satisfied, for $r=p, D=M, V=K, D_{1}=W, D_{2}=C_{M}(W / K)$, and $H=H_{k+1}$.

Proof. (1) follows from Lemma 6.1(e). (2) follows from Lemma 5.2(a). (3) follows from the preceding lemma. (4) and (5) follow from the definitions. (6) follows from Lemma 6.3(c). Hence this lemma is proved.

Lemma 6.16. A contradiction.

Proof. By Lemma 6.15, and Proposition 3.13(a), (b), (c) and (d), 
there exists a subfamily $\Re=\left\{L_{i} ; 1 \leqq i \leqq t\right\}$ of $\widetilde{\mho}_{\sigma}(S)$ which satisfies the following (6.16.1), (6.16.2) and (6.16.3):

$\langle L \in \Re\rangle$ is a solvable $\{2, p\}$-group with a Sylow 2-subgroup $S$ which contains $H_{k+1}$;

there exists a subgroup $T$ of $S$ which is normal in (6.16.2) $\left\langle N_{M}(S), L \in \Re\right\rangle$, and $H_{k+1} / T$ is $p$-closed, and $L / T$ is $p$-closed for all $L \in \Re$;

$$
\text { for each } L \in \Re, O^{2}(L) \cong W \text { or } O^{2}(L) \subseteq C_{M}(W / K) \text {. }
$$

First, we shall show that:

$$
O_{S}\left(\mathfrak{S}_{k+1} \cup\{L\}\right) \supset 1 \text { for all } L \in \Re \text {. }
$$

Suppose that $O_{S}\left(\mathfrak{S}_{k+1} \cup\{L\}\right)=1$ (namely, $\mathfrak{F}_{k+1} \cup\{L\}$ lies in $\mathscr{C} \mathscr{L}^{2}(G)$ ) for some $L \in \Re$. If $O^{2}(L) \leqq C_{M}(W / K)$, then $\left[O^{2}(L), O_{S}(L)\right] \leqq C_{S}(W / K) \leqq$ $O_{S}\left(H_{k}\right)$, which contradicts Lemma $5.13(\mathrm{c})$. So, we get $O^{2}(L) \cong W$ by (6.16.3). Then by Lemma 6.1(b), $\left[O^{2}(L), \Omega_{1} Z \widehat{J}\left(O_{S}\left(\alpha\left(\mathscr{H}_{\mathcal{C}}: H_{j}\right)\right) J_{e}(S)\right)\right]=1$. Then by Lemma $5.20, \delta\left(\mathfrak{S}_{k+1} \cup\{L\}\right)>\delta(\mathfrak{S})$, which contradicts Assumption (A.1). Hence, we obtain (6.16.4). Since $N_{M}(S)=N_{G}(S)$, by (6.16.4), (6.16.2) and Lemma 5.15,

$$
O_{S}\left(\mathfrak{S}_{k+1} \cup \Re\right) \supset 1 \text {. }
$$

Let $F=\langle L \in \Re\rangle$. Then by (6.16.1), $O(L) \subseteq O(F)$ for all $L \in \Re$. Therefore, $F=N_{k^{\prime}}\left(O_{S}\left(\mathfrak{S}_{k+1} \cup \Re\right)\right) O(F)$. Since $H_{k+1} \subseteq F$ by (6.16.1), it follows that

$$
H=N_{I l_{k+1}}\left(O_{S}\left(H_{k+1} \cup \Re\right)\right) O\left(H_{k+1}\right) .
$$

By (6.16.5) and (6.16.6), $1 \subset O_{S}\left(\mathfrak{S}_{k+1} \cup \Re\right) \subseteq O_{S}\left(\mathfrak{S}_{\mathcal{E}}\right)$, which is a final contradiction. This completes the proof of the theorem.

Added in proof. The author would like to give thanks to Professor George Glauberman who pointed out some errors in this paper, and to the Journal for fixing up the manuscript.

\section{REFERENCES}

1. W. Feit and J. G. Thompson, Solvability of groups of odd order, Pacific J. Math., 13 (1963), 775-1029.

2. G. Glauberman, On the automorphism group of a finite group having no nonidentity normal sub- groups of odd order, Math. Z., 93 (1966), 154-160.

3. — A characteristic subgroup of a p-stable groups, Canad. J. Math., 20 (1968). 4. - Global and local properties of finite groups, "Finite Simple Groups, edited by M. B. Powell and G. Higman, Academic Press, 1971. 
5. - Factorizations for 2-constrained groups, to appear.

6. - Factorizations in Local Subgroups in Finite Groups, Amer. Math. Soc. CBMS No. 33, 1978.

7. D. M. Goldschmidt, 2-Fusion in finite groups, Annals of Math., 99 (1974), 70-117.

8. D. Gorenstein, Finite Groups, Harper and Row, New York, 1968.

9. M. Hayashi, 2-Factorization in finite groups, II., in preparation.

10. J. G. Thompson, Normal p-complements for finite groups, J. Algebra, 1 (1964), 43-46.

11. _ - Factorizations of p-solvable groups, Pacific J. Math., 16 (1966), 371-372.

Received April 10, 1978 and in revised form December 14, 1978. This paper was written in partial fulfillment of the requirements of a doctorial thesis at the University of Hokkaido.

Aichi University of Education

Hirosawa, Igaya-Cho, Kariya

JAPAN 448 


\section{PACIFIC JOURNAL OF MATHEMATICS}

\section{EDITORS}

DONALD BABBITT (Managing Editor)

University of California

Los Angeles, California 90024

Hugo RossI

University of Utah

Salt Lake City, UT 84112

C. C. MOORE and ANDrew OGG

University of California

Berkeley, CA 94720
J. DUgundjI

Department of Mathematics University of Southern California Los Angeles, California 90007

R. FinN aNd J. Milgram Stanford University Stanford, California 94305

\section{ASSOCIATE EDITORS}

E. F. BECKENBACH

B. H. NeUMANN

F. WOLF

K. YosHIDA

\section{SUPPORTING INSTITUTIONS}

UNIVERSITY OF BRITISH COLUMBIA CALIFORNIA INSTITUTE OF TECHNOLOGY UNIVERSITY OF CALIFORNIA MONTANA STATE UNIVERSITY UNIVERSITY OF NEVADA, RENO NEW MEXICO STATE UNIVERSITY OREGON STATE UNIVERSITY UNIVERSITY OF OREGON
UNIVERSITY OF SOUTHERN CALIFORNIA STANFORD UNIVERSITY UNIVERSITY OF HAWAII UNIVERSITY OF TOKYO UNIVERSITY OF UTAH WASHINGTON STATE UNIVERSITY UNIVERSITY OF WASHINGTON 


\section{Pacific Journal of Mathematics}

\section{Vol. 84 , No. 1 \\ May, 1979}

Michael James Beeson, Goodman's theorem and beyond ...............

Robert S. Cahn and Michael E. Taylor, Asymptotic behavior of multiplicities

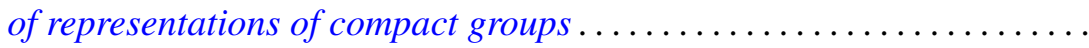

Douglas Michael Campbell and Vikramaditya Singh, Valence properties of

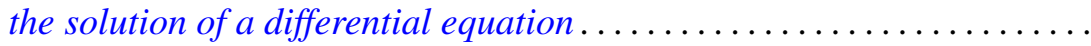

J.-F. Colombeau, Reinhold Meise and Bernard Perrot, A density result in spaces of Silva holomorphic mappings .....................

Marcel Erné, On the relativization of chain topologies .................

Le Baron O. Ferguson, Uniform and $L_{p}$ approximation for generalized

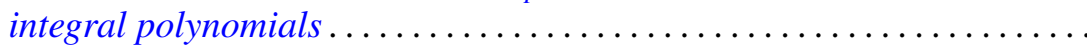

Kenneth R. Goodearl and David E. Handelman, Homogenization of regular rings of bounded index..................................

Friedrich Haslinger, A dual relationship between generalized

Abel-Gončarov bases and certain Pincherle bases .................

Miriam Hausman, Generalization of a theorem of Landau. .

Makoto Hayashi, 2-factorization in finite groups

Robert Marcus, Stochastic diffusion on an unbounded domain ........... 143

Isabel Dotti de Miatello, Extension of actions on Stiefel manifolds....

C. David (Carl) Minda, The hyperbolic metric and coverings of Riemann surfaces...

Somashekhar Amrith Naimpally and Mohan Lal Tikoo, On $T_{1}$-compactifications.

Chia-Ven Pao, Asymptotic stability and nonexistence of global solution for a semilinear parabolic equation ....

Shigeo Segawa, Harmonic majoration of quasibounded type ...

Sze-Kai Tsui and Steve Wright, The splitting of operator algebras ...

Bruce Williams, Hopf invariants, localization and embeddings of Poincaré complexes....

Leslie Wilson, Nonopenness of the set of Thom-Boardman maps ...

Alicia B. Winslow, There are $2^{\mathrm{c}}$ nonhomeomorphic continua in $\beta R^{n}-R^{n}$ 